\title{
Systematics of Fission-Product Yields
}

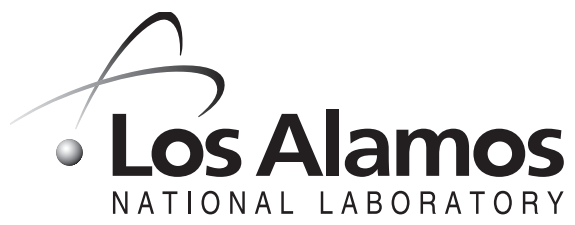

Los Alamos National Laboratory is operated by the University of California for the United States Department of Energy under contract W-7405-ENG-36. 
This work was funded by the US Department of Energy.

An Affirmative Action/Equal Opportunity Employer

This report was prepared as an account of work sponsored by an agency of the United States Government. Neither the Regents of the University of California, the United States Government nor any agency thereof, nor any of their employees make any warranty, express or implied, or assume any legal liability or responsibility for the accuracy, completeness, or usefulness of any information, apparatus, product, or process disclosed, or represent that its use would not infringe privately owned rights. Reference herein to any specific commercial product, process, or service by trade name, trademark, manufacturer, or otherwise does not necessarily constitute or imply its endorsement, recommendation, or favoring by the Regents of the University of California, the United States Government, or any agency thereof. The views and opinions of authors expressed herein do not necessarily state or reflect those of the Regents of the University of California, the United States Government, or any agency thereof. Los Alamos National Laboratory strongly supports academic freedom and a researcher's right to publish; as an institution, however, the Laboratory does not endorse the viewpoint of a publication or guarantee its technical correctness. 
Systematics of Fission-Product Yields

Arthur C. Wahl

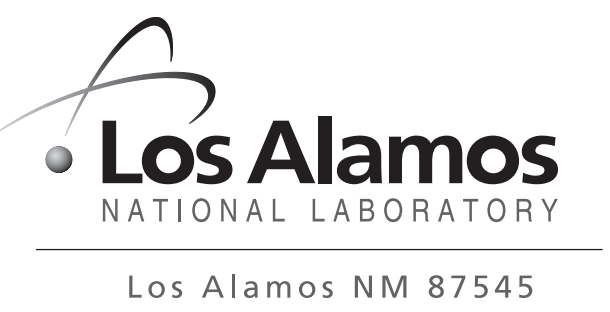





\title{
SYSTEMATICS OF FISSION-PRODUCT YIELDS
}

\author{
Arthur C. Wahl
}

April 25, 2002

\begin{abstract}
Empirical equations representing systematics of fission-product yields have been derived from experimental data. The systematics give some insight into nuclear-structure effects on yields, and the equations allow estimation of yields from fission of any nuclide with atomic number $Z_{F}=90$ thru 98, mass number $A_{F}=230$ thru 252, and precursor excitation energy (projectile kinetic plus binding energies) $P E=0$ thru $\sim 200 \mathrm{MeV}$ - the ranges of these quantities for the fissioning nuclei investigated. Calculations can be made with the computer program CYFP. Estimates of uncertainities in the yield estimates are given by equations, also in CYFP, and range from $\sim 15 \%$ for the highest yield values to several orders of magnitude for very small yield values. A summation method is used to calculate weighted average parameter values for fast-neutron $(\sim$ fission spectrum) induced fission reactions.
\end{abstract}




\section{INTRODUCTION}

Systematics of fission-product yields were derived in two successive stages: (1) Gaussian functions were fitted by the method of least squares to data (mass yields, $Y(A)$, or fractional independent, FI, and cumulative, FC, yields) for each fission reaction investigated. Frequently, not all parameters could be determined by the method of least squares, and some approximate parameter values had to be assumed based on the then current model values. This procedure led to recycling as model values were refined. (2) Each Gaussian parameter determined for a number of fission reactions was fitted to an equation with terms containing differences between $Z_{F}, A_{F}$, and/or $P E$ and corresponding values for U235T [CK61, NE74, WA89, WA99 ${ }^{1}$ using the method of least squares. The equations used and the derived term coefficients are given in Tables 1, 2 and 3, pp. 12, 29 $\& 30$, in the following sections.

It was expedient to treat most low- and high-energy fission reactions separately. Reactions with excitation energies $E^{*}=P E<8.0 \mathrm{MeV}$ were considered low energy, reactions with $P E>20.0 \mathrm{Mev}$ were considered high energy, and reactions with $P E$ between 8.0 and $20.0 \mathrm{MeV}$ were considered intermediate. Gaussian parameters derived from data for the intermediate region, e.g., 14-MeV neutroninduced fission reactions, were used with parameters for both low- and highenergy fission reactions to help achieve smooth model-parameter transitions in going from low- to high-energy.

Data for low-energy fission included yields from spontaneous fission and neutron induced fission with neutron energies of $14-\mathrm{MeV}$ or less. Data for highenergy fission was for proton induced fission reactions, since no yield data were found for high-energy ( $>\sim 14 \mathrm{MeV}$ ) neutron-induced fission. It was assumed that fission yields are independent of how nuclei were formed. The assumption is

\footnotetext{
${ }^{1}$ Reference symbols consist of two letters from the author(s)' names(s) and two numbers for the year. References are listed, pp. 53-57, chronologically.
} 
supported by the observation [FL65] that the peak-to-valley ratio from fission of ${ }^{236} \mathrm{U}$ excited to $11-25 \mathrm{MeV}$ depends only a little $(\sim 13 \%)$ on whether it is formed by neutron capture or by alpha-particle-induced fission of ${ }^{232} \mathrm{Th}$.

Most yield data for high-energy proton-induced fission are presented in the literature as formation cross sections in units of millibarns $(\mathrm{mb})$. In order to use these data with $Y(A)$ and FI - FC used for low-energy fission - cross-section values were divided by the fission cross section and multiplied by 100 to give $Y(A)$ or independent (IN) or cumulative (CU) yields in percent. Then, IN and $\mathrm{CU}$ values were divided by $Y(A)$ to give $\mathrm{FI}$ and $\mathrm{FC}$ values. Fission cross sections and chain yields from the data source were used, if available; otherwise values were deduced from trends in the values with energy. For proton energies above $\sim 25 \mathrm{MeV}$, cross sections for proton induced fission of ${ }^{238} \mathrm{U}$ and ${ }^{232} \mathrm{Th}$ are each approximately constant, $1.40+/-0.10 \mathrm{~b}$, for ${ }^{238} \mathrm{U}$ and $0.89+/-0.06 \mathrm{~b}$ for ${ }^{232} \mathrm{Th}$ [SJ56, SH58, CU63]. Below $\sim 25 \mathrm{MeV}$ there are considerable data useful for interpolation [MC54, FU59, CU63].

For high-energy fission reactions, nucleons are lost before fission, and the initial precursor (target + projectile) values, $P A, P Z$, and $P E$, are lowered (see, for example, [CH82]). The average loss of mass number $(N T)$ and atomic number $(N P E)$ can be determined from yield calculations, but the loss of excitation energy can not, so $P E$ was used as a parameter.

Preliminary calculations showed that a number of parameters used to represent low-energy fission yields, i.e., even-odd proton and neutron factors, $F_{Z}$ and $F_{N}$, slopes of $\sigma_{Z}$ and $\Delta_{Z}$, and special effects near symmetry and on the mass-yield wings, could not be determined from high-energy data, and it was assumed that the effects described by these parameters are negligably small at high energies.

Most of the equations in [WA99] for yields from low-energy fission reactions have been modified to eliminate discontinuities and to enhance smooth transitions of the functions to those for high-energy fission reactions. Equations for 
some Gaussian parameters were derived that can represent both low- and highenergy values. However, for most parameters, different equations were used for low- and high-energy, the two functions being connected by a transition function, linear in $P E$, between $P E=8$ and $20 \mathrm{Mev}$.

It is interesting that $14-\mathrm{MeV}$ neutron-induced fission reactions, treated earlier by low-energy systematics [WA99], are now classified as essentially highenergy reactions. However, the current classification seems appropriate because, as discussed later, neutrons are often emitted before fission induced by $14-\mathrm{MeV}$ neutrons.

Fission yields from fast-neutron-induced fission were calculated by a summation method [WA99] giving weighted average parameter values for those model parameters that are energy dependent. Equations derived for low-energy fission were used (Tables 1 and 2, pp. $12 \& 29$ ) because most of the increments that were summed had $E^{*}$ values $<8 \mathrm{MeV}$. Parameters for U238F were calculated with and without using appropriate values for the intermediate region, and no significant differences in the derived parameter values were observed.

The titles of the sections that follow are:

- Mass Distribution

- Nuclear-charge Distribution

- Models for Neutron Emission

- Fast-neutron-induced Fission Yields

- Calculation of Fission Yields from Systematics - the CYFP Program

- References 


\section{MASS DISTRIBUTION}

\section{Determination of Gaussian Parameters}

Parameters for the multi-Gaussian model [MC74, DI78, WA99] were determined by the method of least squares from experimental chain-yield data for 63 fission reactions. Reciprocal variance weighting was used in the calculations, with minimum errors being set at $5 \%$ for yields from low-energy neutron-induced fission and $10 \%$ for yields from high-energy proton-induced fission reactions. Minimum errors were used to prevent data with very small errors from having unduly large weights for determination of the Gaussian parameters.

The data used were from the two comprehensive evaluations of chain yields [ER93, MI95] for spontaneous fission and for thermal and 14-MeV neutroninduced fission reactions, from yields for monoenergetic-neutron (0.1 to 10.0 $\mathrm{MeV}$ ) induced fission reactions [NF78, GG80, GG81, GG83], from yields for light-wing mass numbers from the LOHENGRIN fission-product separator [DE95, SA89, DI91, HE94, ST96], from LOHENGRIN yields for AM242T $\left({ }^{241} \mathrm{Am}(2 \mathrm{n}, \mathrm{f})\right)$ [MS96], from monoenergetic-proton (10 to $340 \mathrm{MeV}$ ) induced fission of ${ }^{238} \mathrm{U}$ [SH58], and from yields for monoenergetic-proton (13 to $53 \mathrm{MeV}$ ) induced fission of ${ }^{232} \mathrm{Th}$ [CC69]. Mass-yield data for fast-neutron-induced fission can be represented by Gaussian curves, but the Gaussian parameters derived were not used in the second stage of the analysis because fission is induced by neutrons with a large ranges of energies. However, the mass distributions for fast fission reactions can be calculated by a summation method from the model parameters derived from other data, as will be discussed in a later section.

The total number of nucleons emitted $(N T)$, before and after fission, is not generally known for high-energy fission reactions, and values are needed to determine the mid-point (symmetry point, $\bar{A}),(P A-N T) / 2, P A$ being the sum of the projectile and target mass numbers. Therefore, $N T$ was determined as a 
high-energy-fission parameter that gave the best representation of complementarity for light- and heavy-product chain yields for each multi-Gaussian leastsquares calculation. For low-energy fission, $N T=\nu_{F}$, the average total number of post-fission neutrons emitted.

Earlier calculations [WA99] indicated that inclusion of an additional pair of Gaussian curves to help represent experimental $Y(A)$ on the wings of mass-yield curves improved the data representation significantly for some heavier fissioning nuclides (e.g., see: [WA99], Figs. 4.1-3C,4C,5C,6C). Additional calculations using 6 or 7 curves showed that this effect applied to $Z_{F} \geq 94$, but not to $Z_{F} \leq 92$, or to high-energy fission reactions.

\section{Equation for Least-squares Parameter Calculations}

$$
\begin{aligned}
& Y(A)=\sum_{i=1}^{i=7} Y_{i} e^{D} / \sigma_{i} \sqrt{2 \pi} \\
& \\
& D=-\left(A-\bar{A}+\Delta_{i}\right)^{2} / 2 \sigma_{i}^{2} \\
& \bar{A}=(P A-N T) / 2
\end{aligned}
$$

\section{Estimated Model Uncertainties}

It was helpful in efforts to represent measured chain yields by sums of Gaussian curves to have estimates of uncertainties in model calculated yields. Uncertainties were estimated from the following empirical equation proposed earlier for the percent uncertainty $(P E R)$ [WA92].

$$
P E R=25 e^{-0.25 \ln (Y(A))}
$$


Estimated range of uncertainty: $Y(A) /(1+P E R / 100)$ to $Y(A)(1+P E R / 100)$ :

\begin{tabular}{|c|c|c|c|}
\hline Examples: & $Y(A), \%$ & $P E R$ & $1+P E R / 100$ \\
\hline & 10. & 14. & 1.14 \\
\hline & 1. & 25. & 1.25 \\
\hline & 0.1 & 44. & 1.44 \\
\hline & $10^{-3}$ & 141. & 2.41 \\
\hline & $10^{-6}$ & 791. & 8.91 \\
\hline & $10^{-10}$ & 7,910 . & 80.10 \\
\hline & $10^{-15}$ & 140.600 & 1.407 . \\
\hline
\end{tabular}

Examples of results of multi-Gaussian least-squares (LS) calculations for individual fission reactions are shown in Figs. 1a - 10a, pp. 13-17. (Figs. 1b-10b show the same experimental points, but the lines are derived from systematic trends, to be discussed later.) It can be seen that most experimental chain yields (points in the figures) are represented approximately by the sums (solid lines) of several Gaussian curves (broken lines), each sum being normalized to $200 \%$. The dotted lines, between which many data-points fall, represent the estimated uncertainties for the sums of the calculated curves. The reduced Chi-square values in parentheses, which include estimated model uncertainties discussed above, cluster about 1.0, and the average of the 10 values shown in the figures is 1.0. Thus, the estimated uncertainties for the multi-Gaussian representations behave appropriately for the limited test described. The reduced Chi-square values before the parentheses in the figures are based on experimental or minimum errors and are mostly $>1.0$, showing that the multi-Gaussian model does not represent the fine-structure and other details from experiments very well. 


\section{Systematics of Gaussian Parameters}

The parameters determined for the $3-7$ Gaussian functions for each fission reaction were fitted by mathematical functions of the average $Z_{F}$ and $A_{F}$ of fissioning nuclei and $P E$, the precursor excitation energy, using the method of least squares. Reciprocal variance weighting was used with errors determined earlier for each parameter value or with the minimum error of $5 \%$. The functions derived, given in Table 1, p. 12, allow calculation of mass distributions for any fissioning nuclei in the regions investigated.

Chain yield data for proton induced fission of ${ }^{238} \mathrm{U}$ [SH58] (9 data sets with mono-energetic protons from 10 to $340 \mathrm{MeV}$ ) were used to represent high-energy fission reactions, with the assumption that use of protons instead of neutrons had a negligable effect on model parameters. (See Introduction.) Most parameters for the Gaussian curves change fairly rapidly with energy at the lower energies and then level off above $P E=\sim 100 \mathrm{MeV}$, consistent with the similarity of high-energy chain-yield curves [SH58]. In preliminary calculations, data and parameters for proton induced fission of ${ }^{232} \mathrm{Th}$ [CC69] (7 data sets with mono-energetic proton from 13 to $53 \mathrm{MeV}$ ) were included. These data consisted of fragment yields, before neutron evaporation, read from mass-yield curves, without points, and derived from kinetic-energy measurements. The Gaussian parameters obtained were of the same magnitude as those from radiochemically determined fission-product yields from ${ }^{238} \mathrm{U}+p$ [SH58], but the variation of parameters with energy was different. (See Figs. 11-13, p. 18.)

The method of least squares was used to identify the more important terms for each model-parameter function and to determine the best coefficient values for these terms. Only terms making significant contributions to the reduction of reduced $\chi^{2}$ from least-squares calculations were included in an equation. The results are summarized in Table 1, p. 12. Some parameters could be represented by the simpler Eq. 3. The intensity of the central curve, $Y_{3}$, which increases 
with $P E$ by several orders of magnitude (see Fig. 13, p. 18), required different equations for low- and high-energies, as shown in the footnotes to Table 1.

\section{Principal Peak Curves}

This pair of curves is the principal contributor to the mass distribution for both high- and low-energy fission. Equations for $\sigma_{1,5}$ and $\Delta_{1,5}$ were derived that represent both low- and high-energy fission reactions. The equations are dependent on both $Z_{F}$ and $P E$, as shown in Table 1, Eq. 3, p. 12. The energy dependencies are shown in Figs. 11 and 12, p. 18. The intensities of these curves, $Y_{1,5}$, were adjusted (normalized) so that the sum of intensities of all curves was $200 \%$.

\section{Inner Peak Curves}

The yield of the pair of inner peak curves, $Y_{2,4}$, decreases with $P E$ and goes to zero when $P E>\sim 20 \mathrm{MeV}$ as shown in Fig 14, p. 19. The inner peak curves are narrow, as shown in Fig. 15, p. 19, and represent the sharp change in yields that occurs below the heavy mass number, $A_{H}=130$, and above the light complement. (These curves replace the exponential functions used previously [WA99].)

The mass numbers, $A_{4}$, at the maxima of the heavy inner-peak curves, were determined, and a function (Eq. 3, Table 1, p. 12) was fitted to the values by the method of least squares. The resulting parameter values for the function are given in Table 1. The displacements, $\Delta_{2}$ and $\Delta_{4}$, were then calculated from the function using Eq. 5 in the footnotes to Table 1. Figure 16, p. 19, shows the values (points) and the derived function (the line), both corrected for the small $Z_{F}$ dependence to allow comparison of parameter values for a number of fission reactions with the derived function. 
The narrowness of the inner peak curves, a result of the sharp decrease in yields below $A_{H}=\sim 130$, and above the light complementary $A_{L}=P A-130-N T$, as well as the large kinetic energies [BB83] and small prompt neutron emission near $A=130$ [WA88], may all result from the proximities of the $Z$ and $N$ of nascent heavy fragments to the $Z=50$ and $N=82$ spherical shells.

\section{Central Peak Curve}

The maximum for this curve is at the midpoint, $\Delta_{3}=0.0, A_{3}=(P A-N T) / 2$. The width-parameter for the central peak, $\sigma_{3}=8.6+/-1.0$, is the average of 10 values from fission of thorium isotopes. The principal peak separations were larger for these than for other fission reactions investigated, giving optimal information about properties of the central peak. (e.g., see Figs. 1a, b, p. 13, and compare to Figs. 2a, b-10a, b, pp 14-17.) The value of 8.6 better approximates the nearly flat valleys observed for a number of experimental mass-yield data than does the smaller value of 6.0 used previously [WA99]. For high-energy fission reactions, $\sigma_{3}$ was taken to be 8.6 or $\sigma_{1}$, whichever was larger.

The curve intensity, $Y_{3}$, increases several orders-of-magnitude with increasing $P E, \sim 0.015 \%$ from model calculations, for spontaneous fission [WA99] and $\sim 90 \%$ for high-energy fission. This large change required two functions, the two being applied in different regions of $P E$, Eqs. 7 and 8, below Table 1, p. 12, and Fig. 13, p. 18. The transition between functions is quite smooth. (Two exponential functions with different slopes were used in [WA99].)

\section{Wing Curves}

Least-squares calculations for nuclei with $Z_{F} \geq 94$ showed that the wingcurve parameters $Y_{6,7}$ and $\Delta_{7}$ were essentially constant for low $P E(<8 \mathrm{MeV})$, and average values are shown in Table 1, p. 12. $Y_{6,7}$ is assumed to be $3.4 \%$ for $Z_{F}$ $=93$, and $Y_{6,7}$ is assumed to decrease linearly from its value at $E *=P E=8.0$ 
$\mathrm{MeV}$ to 0.0 at $P E=20.0 \mathrm{MeV}$. The widths of the curves increase with $Z_{F}$ as shown for $\sigma_{6,7}$ in Table 1.

\section{Number of Nucleons Emitted}

Neither the original [HO77] nor the revised version [WA99] of Howerton's equation for estimating $\nu_{F}$ was intended to be used for extrapolation to high energies, so the total number of nucleons emitted, $N T$, needed to establish the symmetry point, $(P A-N T) / 2$, was determined as a model parameter. Figure 17 , p. 19, shows $N T$ values determined by least squares from mass-yield data for a number of different nuclides and excitation energies, $P E$, (points) and the function, Eq. (3) in Table 1, p. 12, (the lines) derived to represent these values. There is considerable uncertainty and scatter in values for proton-induced fission, possibly because the mass-yield data [SH58] were limited, although these were the best that were available.

\section{Systematic Trends Represented by Parameter Equations}

The equations and parameter values in Table 1, p. 12, represent systematic trends in the multi-Gaussian model parameters that allow estimation of chain yields from both low- and high-energy fission reactions. Figures 11-17, pp. 18 \& 19 , show that the equations represent most of the parameters derived from the chain yields only moderately well, there being considerable scatter of points.

The reduced $\chi^{2}$ values shown in Figs. 1b-10b, pp. 13-17, are mostly greater than one, showing that the models do not represent most data within a minimal 5 or $10 \%$ error or larger experimental error. Many of the recalculated reduced $\chi^{2}$ values, that include estimated model uncertainties, shown in parentheses, are $>1.0$, indicating that the estimated model uncertainties are too small. Perhaps the coefficient of 25 in Eq. 1 should be increased for yields derived from equations representing systematics shown in Table 1, p. 12. 


\section{TABLE 1 - EQUATIONS FOR SYSTEMATIC TRENDS IN GAUSSIAN PARAMETERS FOR Y(A)}

$$
\begin{aligned}
\text { Par. }=P 1+(P 2-P 1)\left(1.0-e^{P 3 * P E}\right) & \\
P 1 & =P(1)+P(4)\left[Z_{F}-92\right] \\
P 2 & =P(2)+P(5)\left[Z_{F}-92\right] \\
P 3 & =P(3)+P(6)\left[Z_{F}-92\right]
\end{aligned}
$$

Par. $=P 1+P 2 * P E$

\begin{tabular}{lcccccccc}
\hline Par. & $E q$. & $P(1)$ & $P(2)$ & $P(3)$ & $P(4)$ & $P(5)$ & $P(6)$ & No. $^{a}$ \\
\hline$\sigma_{1,5}$ & 3 & 2.808 & 8.685 & -0.0454 & 0.372 & -0.620 & -0.0122 & 63 \\
$\sigma_{2,4}$ & 4 & 2.45 & 0.0 & - & 0.0 & 0.0 & - & 38 \\
$\sigma_{3}{ }^{b}$ & 4 & 8.6 & 0.0 & - & 0.0 & 0.0 & - & 10 \\
$\sigma_{6,7}$ & 4 & 3.17 & 0.0 & - & 0.303 & 0.0 & - & 6 \\
$\Delta_{5}{ }^{c}$ & 3 & 25.34 & 18.55 & -0.0402 & -1.220 & -1.732 & 0.0 & 63 \\
$A_{4}{ }^{d}$ & 4 & 136.66 & -0.177 & - & 0.060 & -0.038 & - & 45 \\
$\Delta_{7}{ }^{c}$ & 4 & 30.31 & 0.0 & - & 0.0 & 0.0 & - & 5 \\
$Y_{2,4}{ }^{e}$ & 4 & 43.00 & -1.91 & - & -3.41 & 0.0 & - & 47 \\
$Y_{6,7}{ }^{f}$ & 4 & 6.80 & 0.0 & - & 0.0 & 0.0 & - & 10 \\
$N T$ & 3 & 1.563 & 16.66 & -0.00804 & 0.0918 & 0.0 & 0.0 & 45 \\
\hline
\end{tabular}

${ }^{a}$ Number of parameter values used in least-squares calculation.

${ }^{b} \sigma_{3}=\sigma_{1,5}$ if $\sigma_{1,5}>8.6$

${ }^{c} \Delta_{1}=-\Delta_{5}, \Delta_{6}=-\Delta_{7}$

${ }^{d} \Delta_{4}=A_{4}-\left(A_{F}-N T\right) / 2.0, \Delta_{2}=-\Delta_{4}$

${ }^{e}$ If $Y_{2,4}<0.0, Y_{2,4}=0.0$

${ }^{f}$ If $P E>8 \mathrm{MeV}, Y_{6,7}=6.8-(6.8 / 12) *(P E-8.0)$

$$
\begin{aligned}
& \text { If } Z_{F}=93, Y_{6,7}=Y_{6,7} / 2.0 \\
& \text { If } Z_{F}<93 \text { or } P E>20 \mathrm{MeV}, Y_{6,7}=0.0
\end{aligned}
$$

$$
Y_{3}=4.060 e^{[0.470(P E-11.96)]} \text { if } P E<11.96
$$

$$
\begin{array}{r}
Y_{3}=4.060+86.02\left(1.0-e^{T(P E-11.96)}\right) \text { if } P E \geq 11.96 \text { No. }^{a}=63 \\
T=-0.030+0.0050\left(A_{F}-236.0\right)
\end{array}
$$



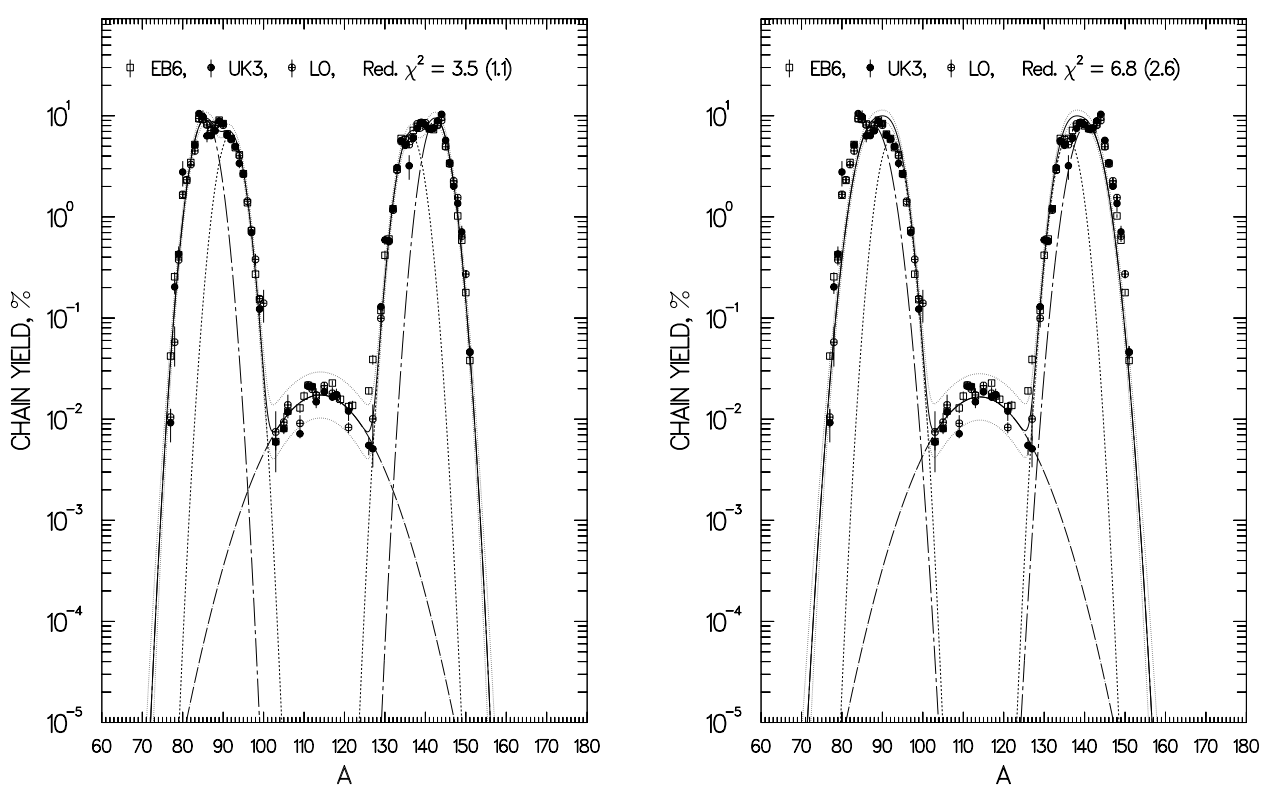

Fig. 1a - TH229T, L.S. Par., 5\% min. error

Fig. 1b-TH229T, Sys. Par., 5\% min. error
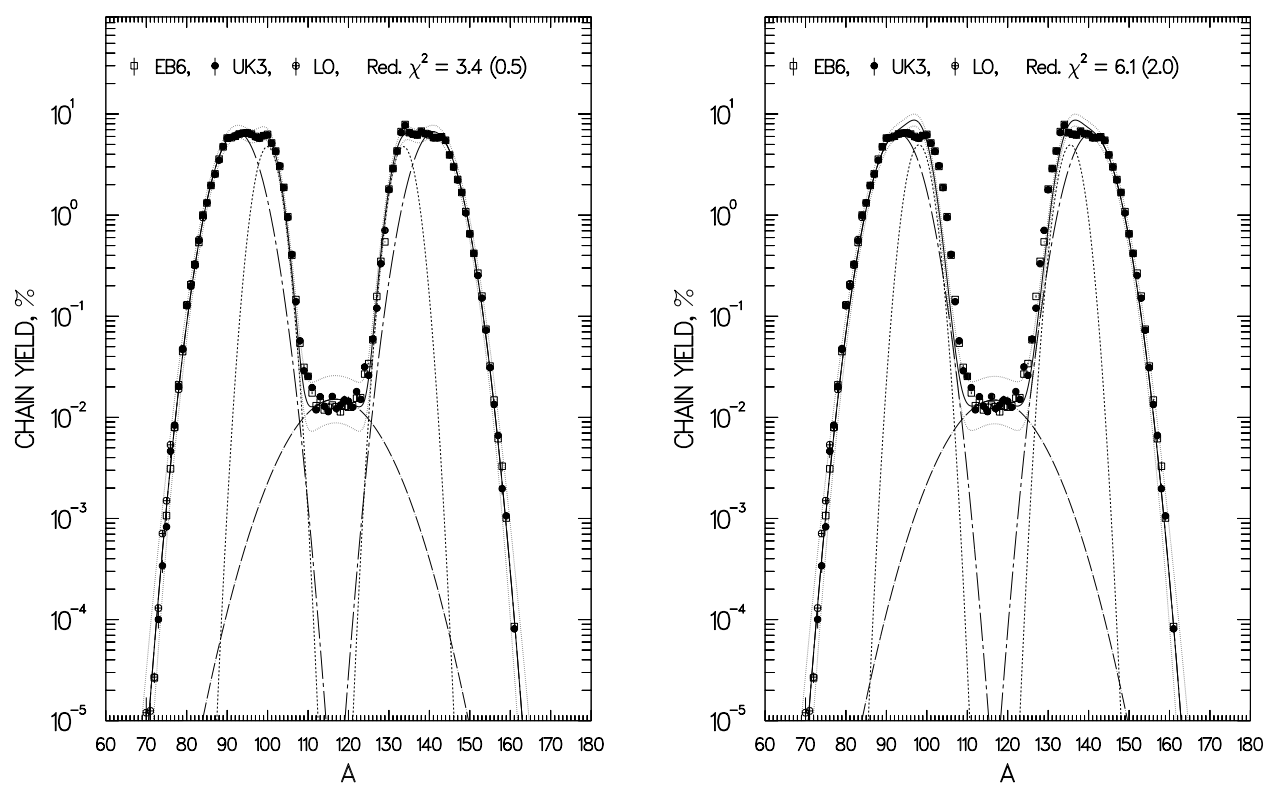

Fig. 2a-U235T, L.S. Par., 5\% min. error

Fig. 2b-U235T, Sys. Par., 5\% min. error 


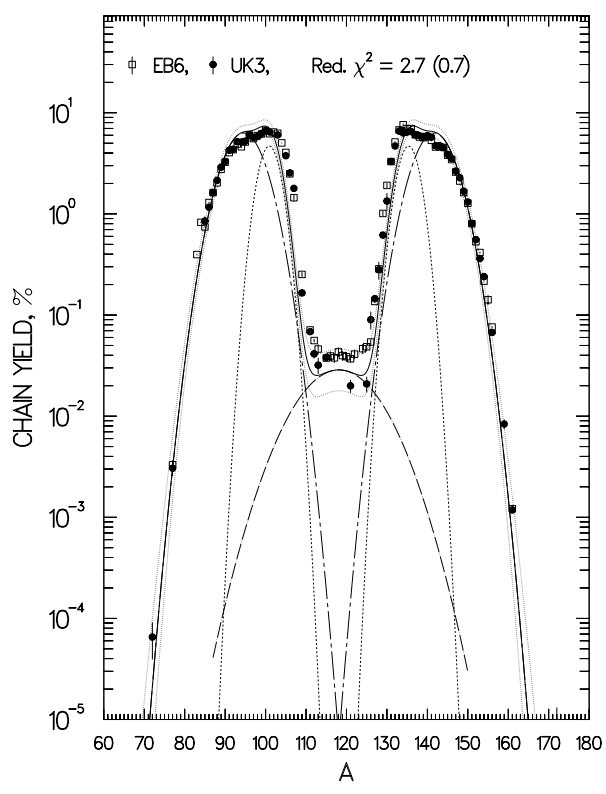

Fig. 3a-U238F, L.S. Par., 5\% min. error

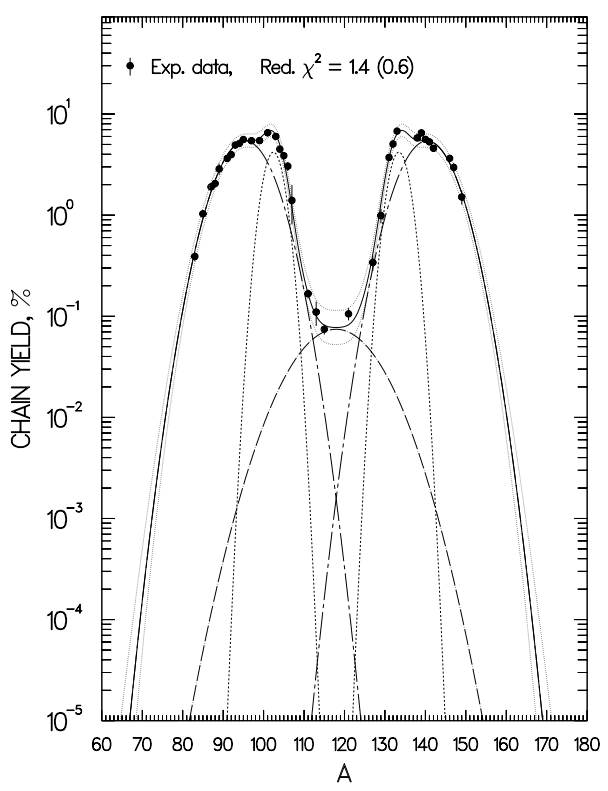

Fig. $4 \mathrm{a}-{ }^{238} \mathrm{U}+5.5 \mathrm{MeV}$ n, L.S. Par.

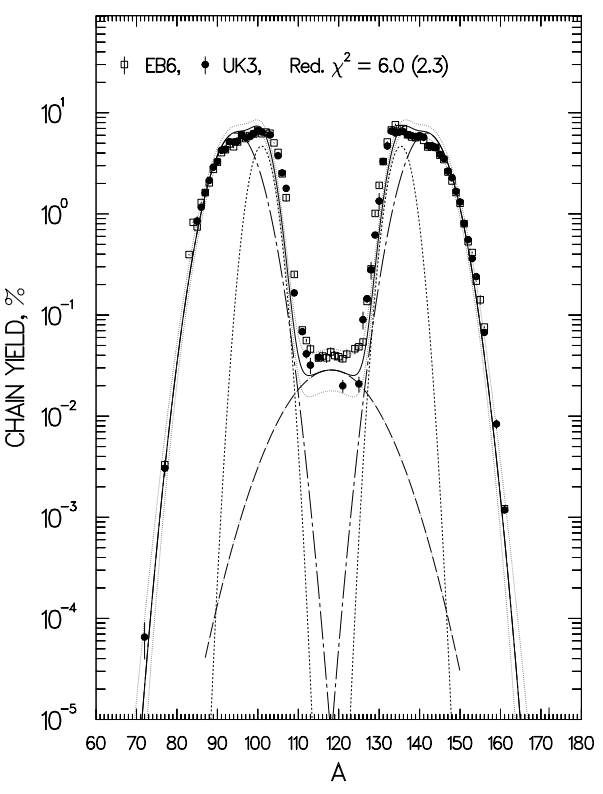

Fig. 3b-U238F, Sys. Par., 5\% min. error

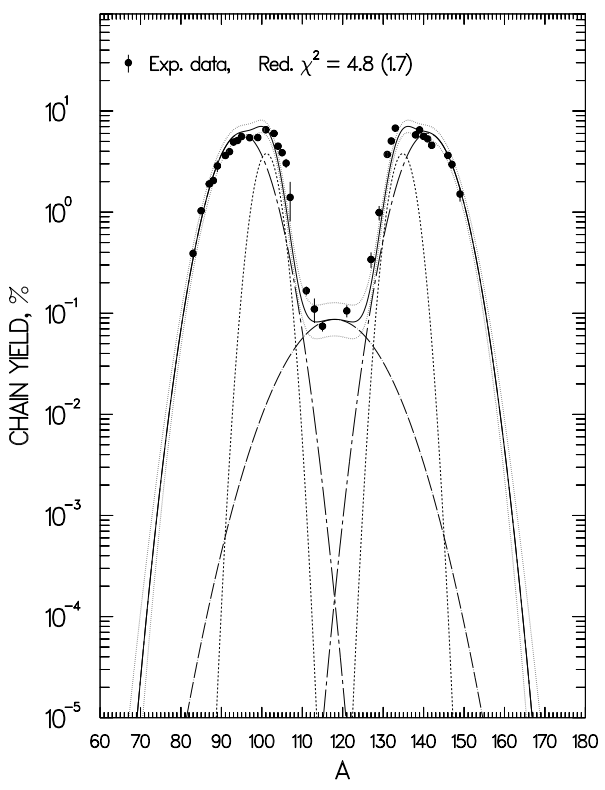

Fig. $4 \mathrm{~b}-{ }^{238} \mathrm{U}+5.5 \mathrm{MeV}$ n, Sys. Par. 


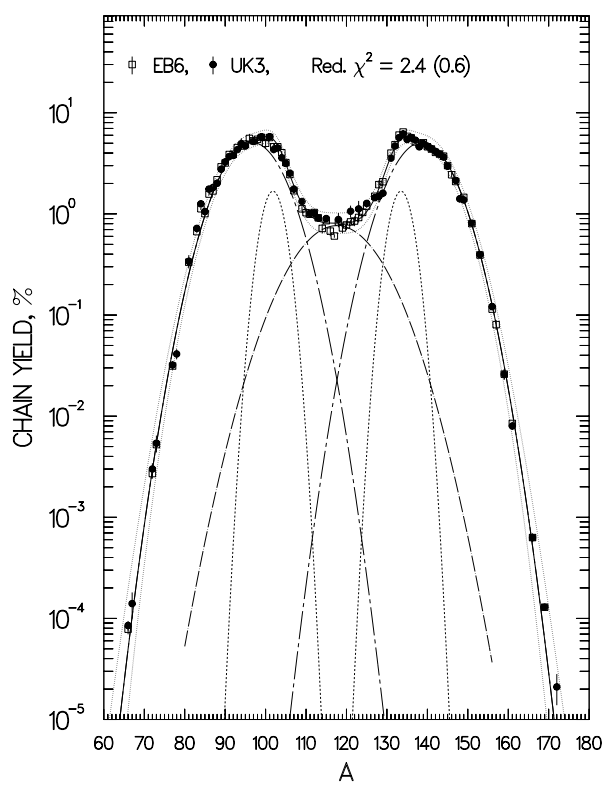

Fig. 5a-U238H, L.S. Par., 5\% min. error

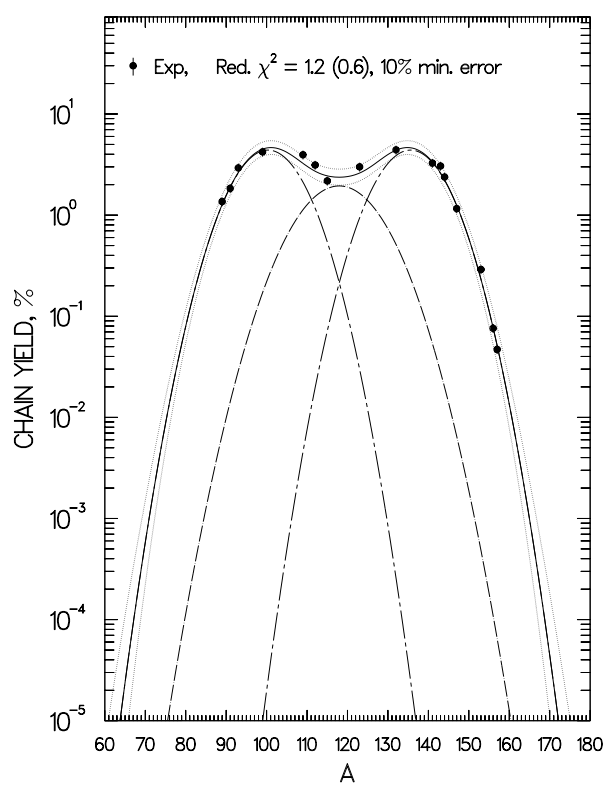

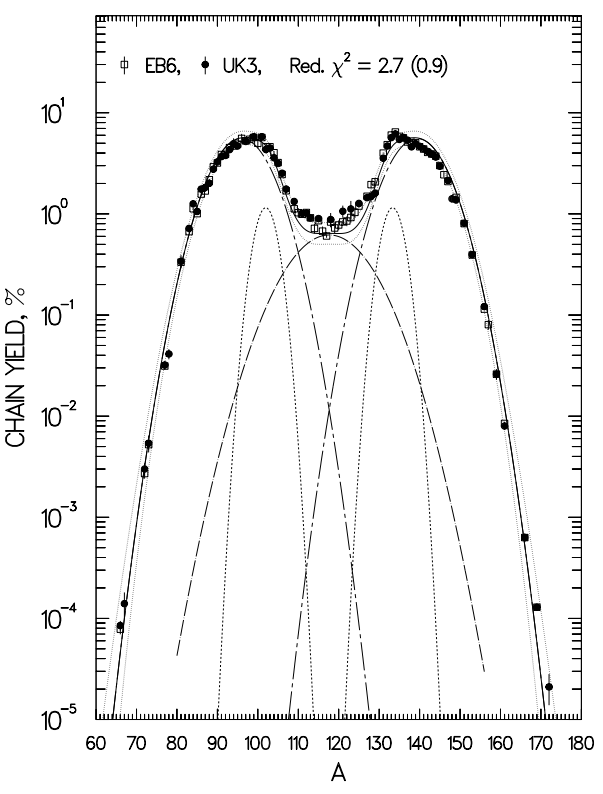

Fig. 5b-U238H, Sys. Par., 5\% min. error

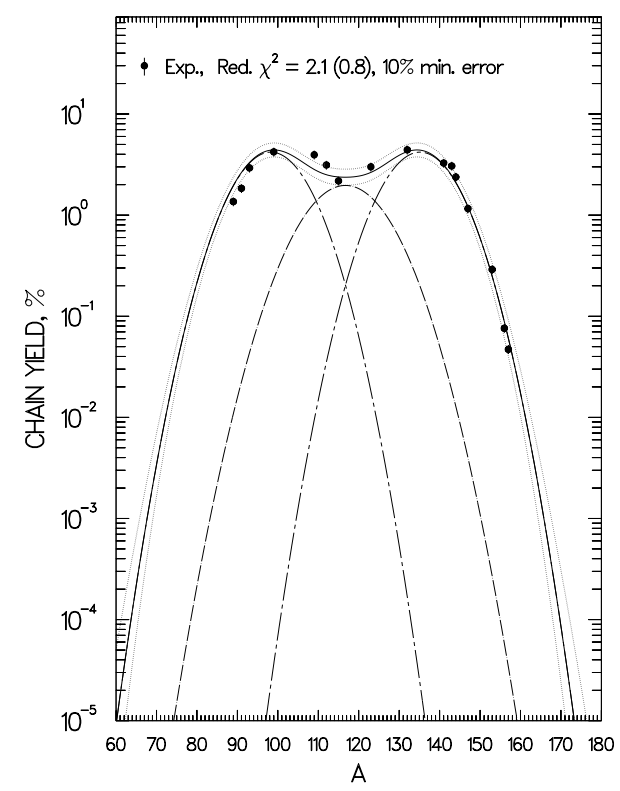

Fig. 6a-U238 + $32 \mathrm{MeV}$ p, L.S. Par. Fig. 6b-U238 + $32 \mathrm{MeV}$ p, Sys.Par. 


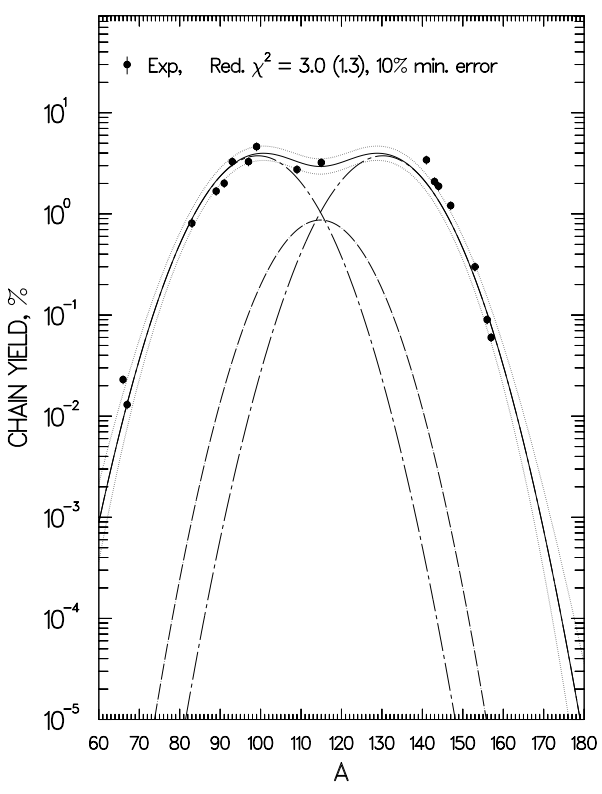

Fig. $7 \mathrm{a}-\mathrm{U} 238+100 \mathrm{MeV}$ p, L.S. Par.

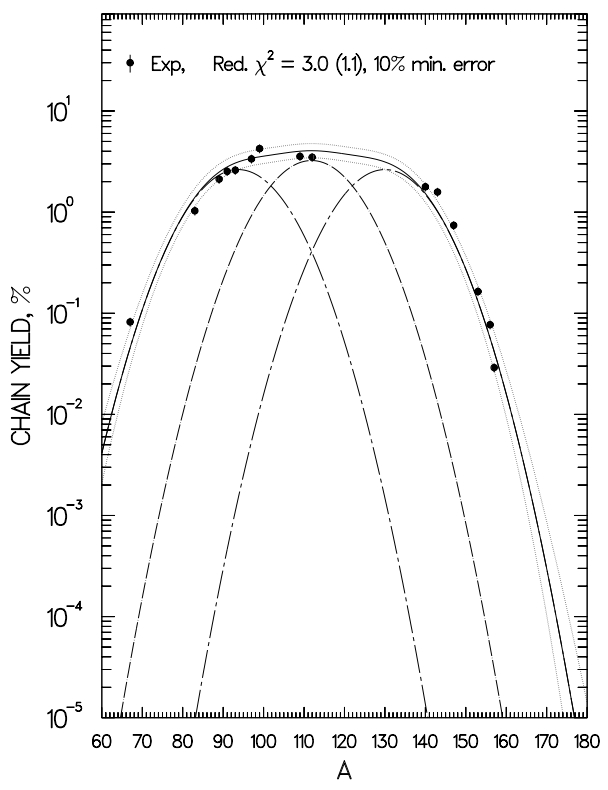

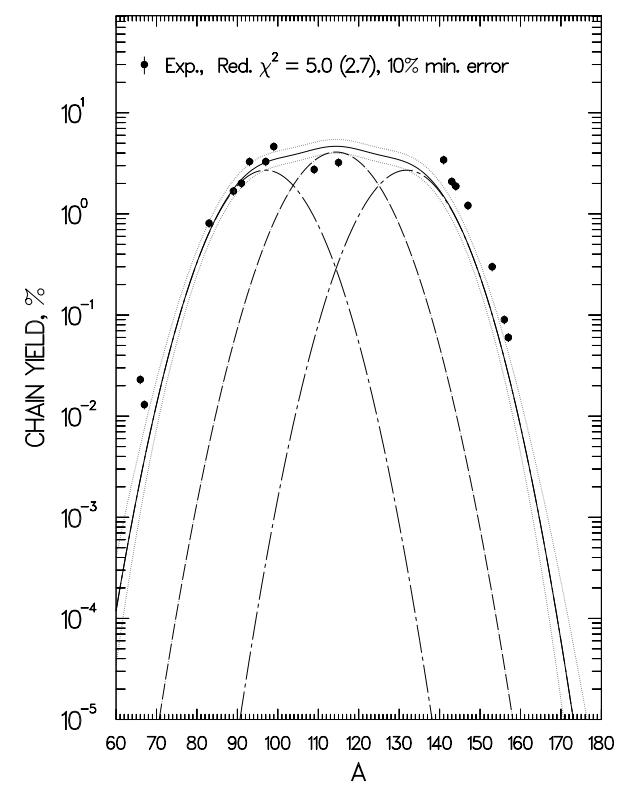

Fig. $7 \mathrm{~b}-\mathrm{U} 238+100 \mathrm{MeV}$ p, Sys. Par.

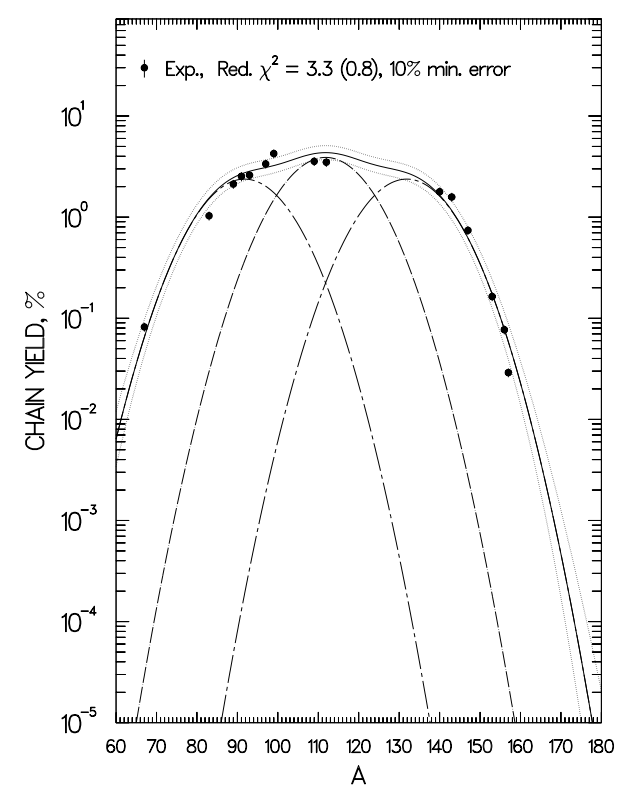

Fig. $8 \mathrm{a}-\mathrm{U} 238+300 \mathrm{MeV}$ p, L.S. Par. $\quad$ Fig. $8 \mathrm{~b}-\mathrm{U} 238+300 \mathrm{MeV}$ p, Sys.Par. 

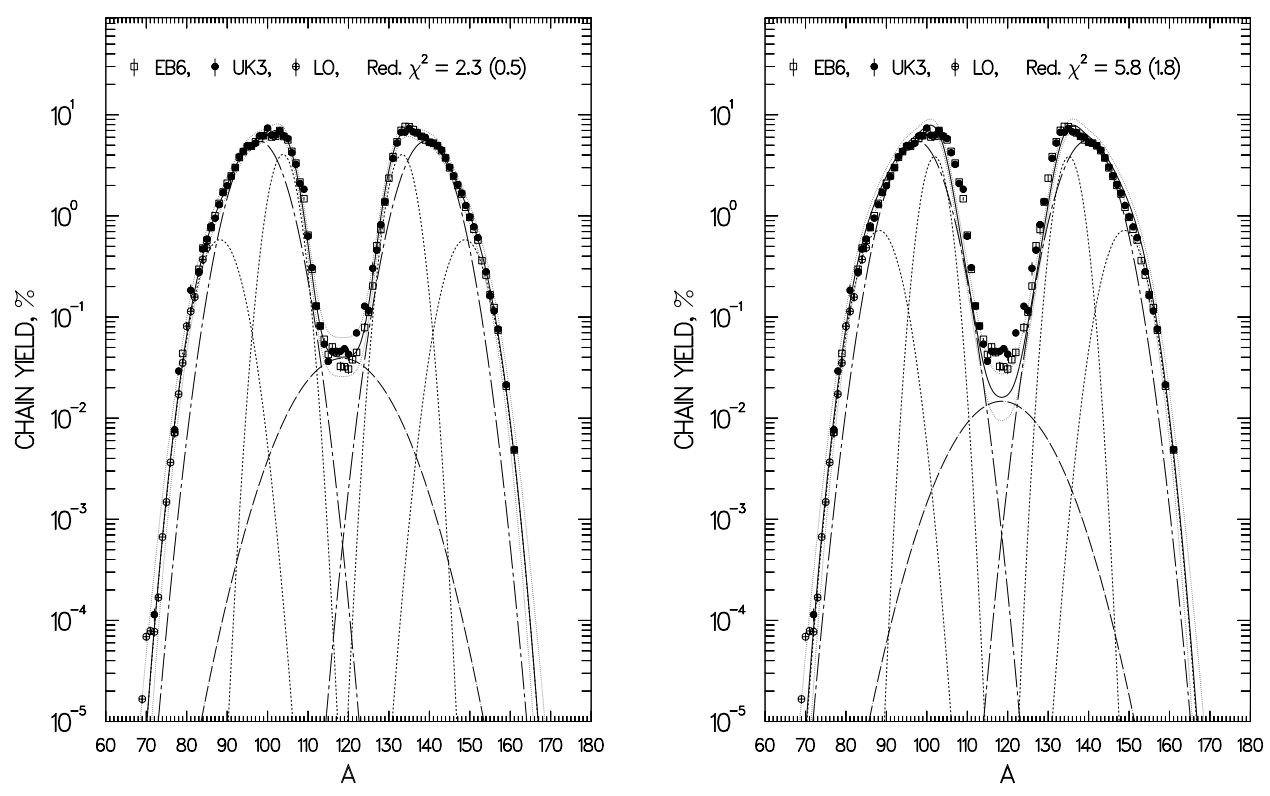

Fig. 9a-PU239T, L.S. Par., 5\% min. error Fig. 9b-PU239T, Sys. Par., 5\% min. error
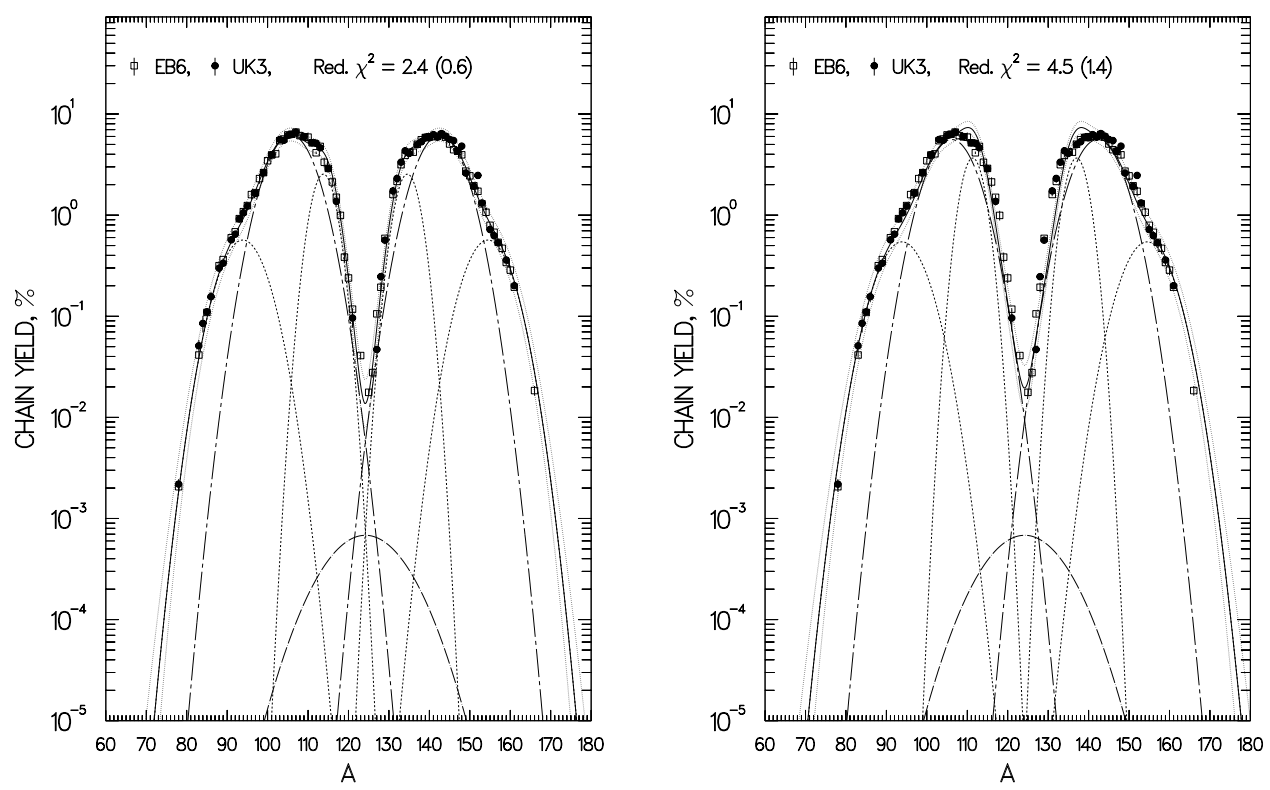

Fig. 10a-CF252S, L.S. Par., 5\% min. error

Fig. 10b-CF252S, Sys. Par., 5\% min. error 


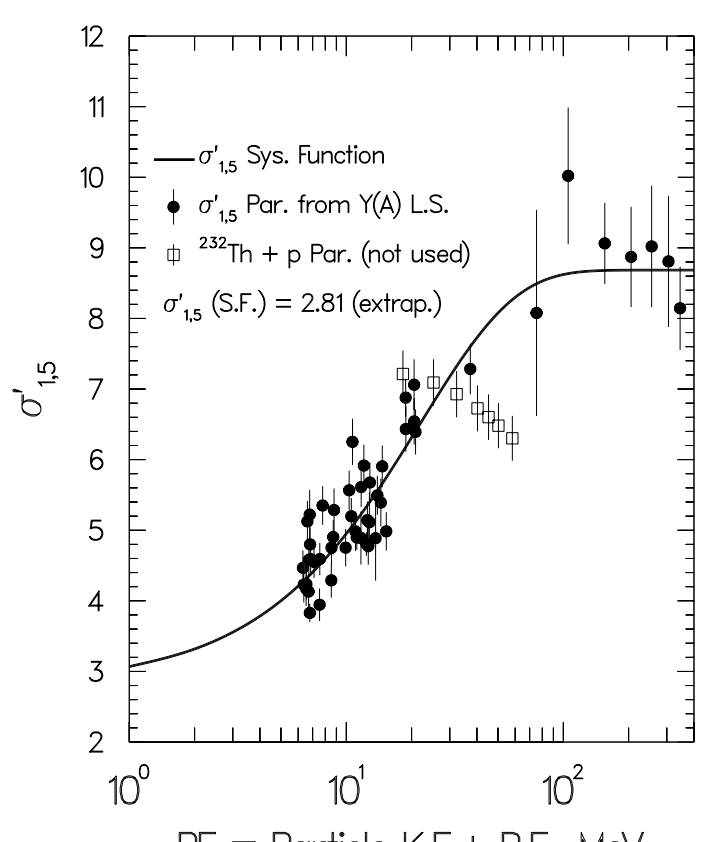

$P E=$ Particle K.E.+ B.E., MeV

Fig. $11-\sigma_{1,5}^{\prime}$ Function, $\sigma_{1,5}^{\prime}=\sigma_{1,5}-\mathrm{f}\left(\mathrm{Z}_{F}\right)$

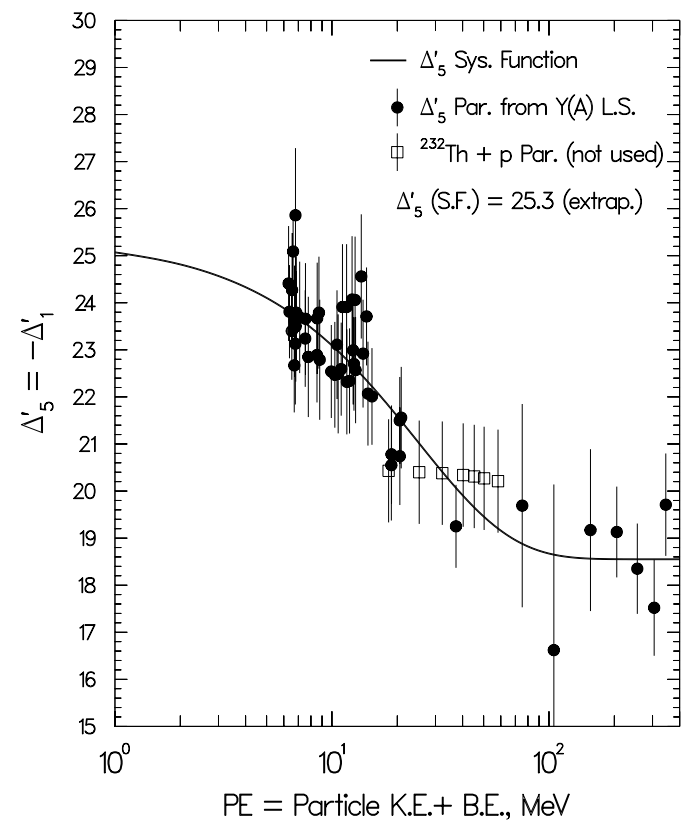

Fig. $12-\Delta_{5}^{\prime}$ Function, $\Delta_{5}^{\prime}=\Delta_{5}-\mathrm{f}\left(\mathrm{Z}_{F}\right)$

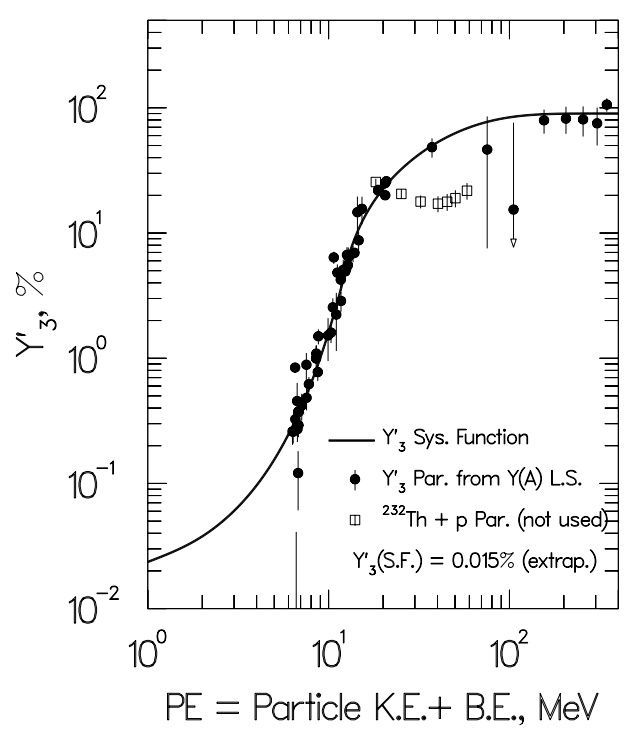

Fig. $13-\mathrm{Y}_{3}^{\prime}$ Function, $\mathrm{Y}_{3}^{\prime}=\mathrm{Y}_{3}-\mathrm{f}\left(\mathrm{A}_{f}\right)$ 


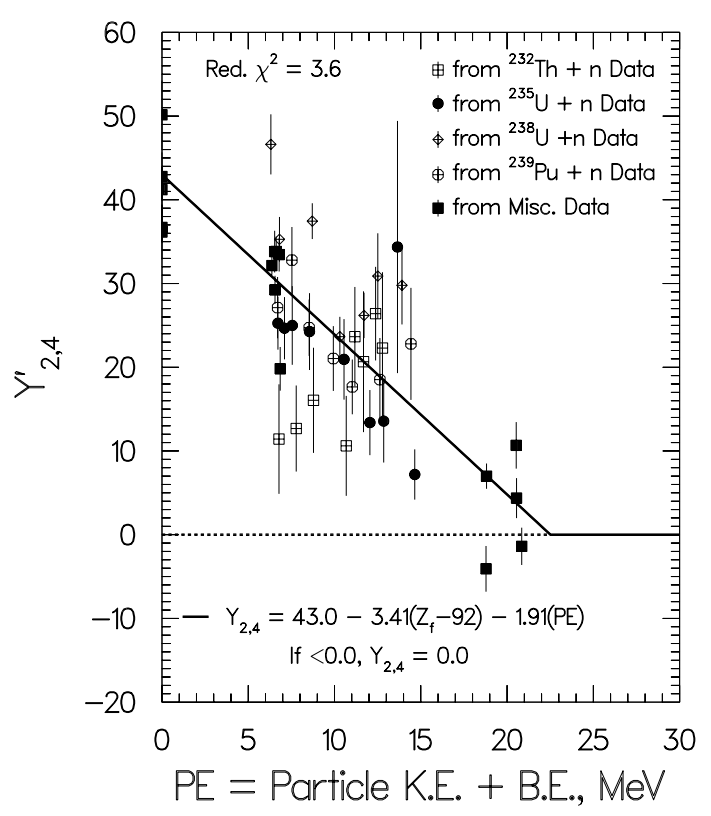

Fig. $14-\mathrm{Y}_{2,4}^{\prime}=\mathrm{Y}_{2,4}-\mathrm{f}\left(\mathrm{Z}_{f}\right)$ Function

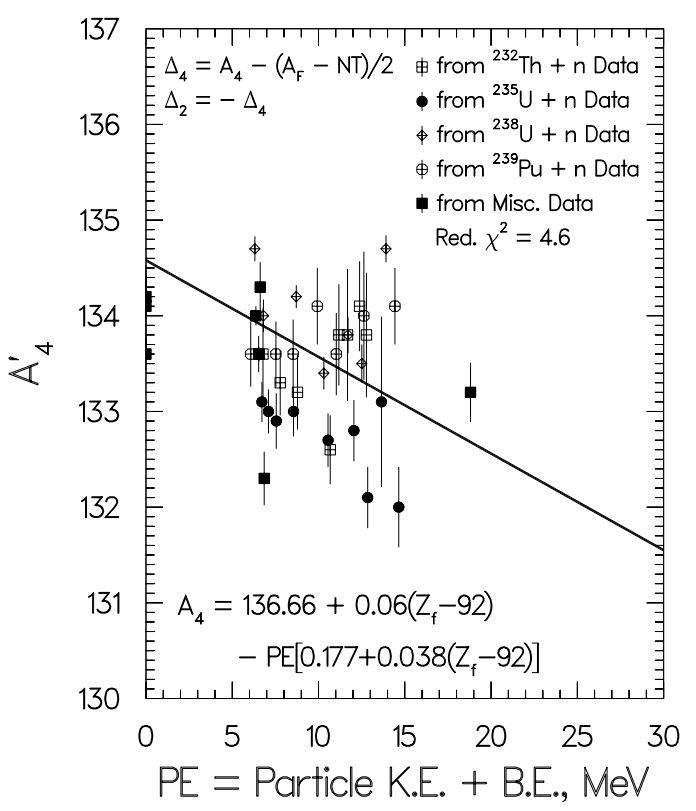

Fig. $16-\mathrm{A}_{4}^{\prime}=\mathrm{A}_{4}-\mathrm{f}\left(\mathrm{Z}_{f}\right)$ Function

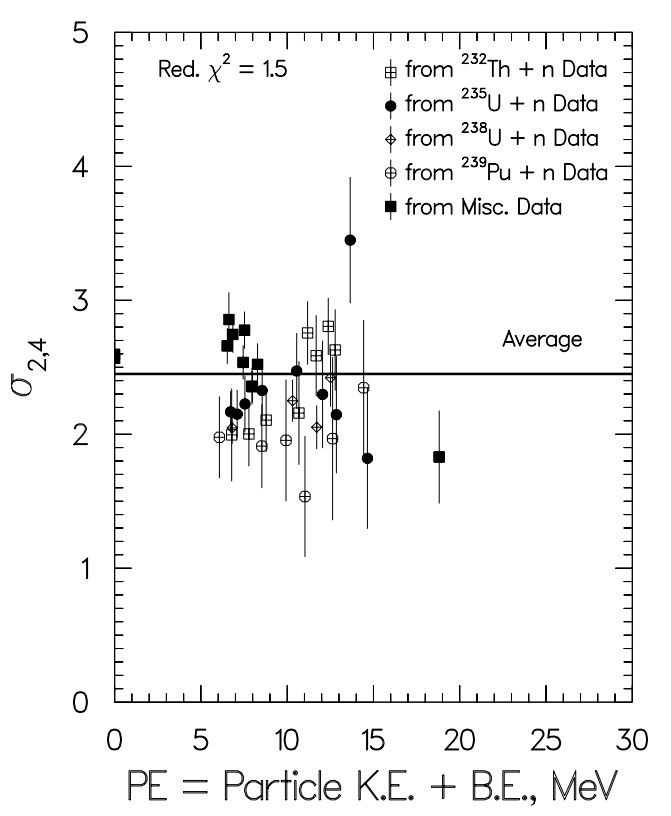

Fig. 15 - Average $\sigma_{2,4}=2.45 \pm 0.05$

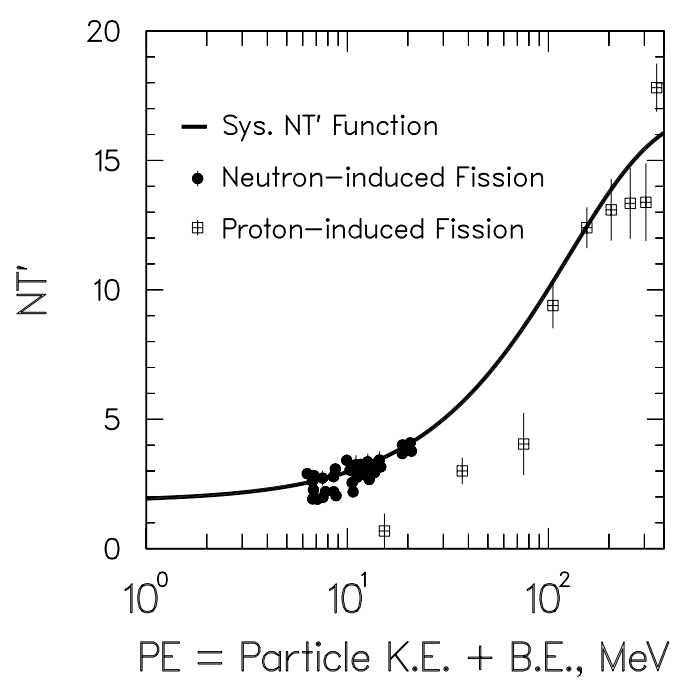

Fig. $17-\mathrm{NT}^{\prime}=\mathrm{NT}-\mathrm{f}\left(\mathrm{Z}_{f}\right)$ Function 


\section{NUCLEAR-CHARGE DISTRIBUTION}

Nuclear-charge distribution describes the dispersion of yields with mass and atomic numbers, $A$ and $Z$, of more than a 1000 primary fission products from each of many fission reactions. The yields are for products after prompt neutron emission and before beta decay. Since only a small fraction of the yields have been measured, models are needed for estimation of unmeasured yields. Theoretical models are not sufficiently advanced to give reliable yield estimates, so the empirical $Z_{P}$ model [WA88, WA99] has been used.

The $Z_{P}$ model treats dispersion of fractional independent yields, FI, of primary fission products with $\mathrm{Z}$ for each A. Gaussian dispersion, modified for even-odd proton and neutron effects, is assumed. Parameters for the model were determined by the method of least squares from fractional independent (FI) and fractional cumulative (FC) yield values derived from experimental data. Parameter values for 25 fission reactions with excitation energies from $0 \mathrm{MeV}$ (spontaneous fission) to about $170 \mathrm{MeV}$ (proton-induced fission) were used.

\section{Equations for the $Z_{P}$ Model}

The equations for the $Z_{P}$ model [WA88], given below, involve the error function of $\mathrm{x}, \operatorname{erf}(\mathrm{x})$. Complementarity of light and heavy fission products is approximated by $A^{\prime}=A+\nu_{P}(A)$ and $A_{L}^{\prime}+A_{H}^{\prime}=A_{F}$ to allow the same or complementary functions to be used for both light and heavy products. Determination of the $\nu_{P}(A)$ function, the average number of post fission neutrons emitted to form fission products with $A$, is discussed in the next section. The parameters $\sigma_{Z}, \Delta_{Z}, F_{Z}, F_{N}$ and their slopes with respect to $A^{\prime}$ were determined in contiguous regions of $A^{\prime}$ for each fission reaction by the method of least squares. 


$$
\begin{gathered}
F I(A, Z)=[0.5][F(A)][N(A)][\operatorname{erf}(V)-\operatorname{erf}(W)] \\
V=\frac{Z(A)-Z_{P}(A)+0.5}{\sigma_{Z}\left(A^{\prime}\right) \sqrt{2}} \\
W=\frac{Z(A)-Z_{P}(A)-0.5}{\sigma_{Z}\left(A^{\prime}\right) \sqrt{2}} \\
Z_{P}\left(A_{H}\right)=A_{H}^{\prime}\left[Z_{F} / A_{F}\right]+\Delta Z\left(A_{H}^{\prime}\right) \\
Z_{P}\left(A_{L}\right)=A_{L}^{\prime}\left[Z_{F} / A_{F}\right]-\Delta Z\left(A_{H c}^{\prime}\right),\left(A_{H c}^{\prime}=A_{F}-A_{L}^{\prime}\right)
\end{gathered}
$$

$$
\begin{aligned}
& F(A)=\left[F_{Z}\left(A^{\prime}\right)\right]\left[F_{N}\left(A^{\prime}\right)\right] \quad \begin{array}{lll}
\underline{\text { for } Z} & \underline{\text { for } N} \\
\text { even } & \text { even }
\end{array} \\
& F(A)=\left[F_{Z}\left(A^{\prime}\right)\right] /\left[F_{N}\left(A^{\prime}\right)\right] \quad \text { even } \quad \text { odd } \\
& F(A)=\left[F_{N}\left(A^{\prime}\right)\right] /\left[\left(F_{Z}\left(A^{\prime}\right)\right] \quad \text { odd } \quad\right. \text { even } \\
& F(A)=1 /\left[F_{Z}\left(A^{\prime}\right)\right]\left[F_{N}\left(A^{\prime}\right)\right] \quad \text { odd } \quad \text { odd }
\end{aligned}
$$

The normalization factor, $N(A)$, applied to achieve $\sum(\mathrm{FI})=1.00$ for each $A$, is required because the even-odd factors, $F(A)$, destroy the inherent normalization properties of Gaussian distributions. Values of $N(A)$ seldom deviate by more than $10 \%$ from unity.

The minimum error for low-energy fission, used for reciprocal variance weighting, was set to 0.1 to prevent very small $\mathrm{FI}$ and $\mathrm{FC}$ values with very small absolute errors from being given unduely high weights, but still having weights similar to those of high-yield products. For high-energy fission the larger of the experimental error, 0.01 or $10 \%$ of a value, was used for weighting. Values of 
$F_{Z}\left(A^{\prime}\right)$ and $F_{N}\left(A^{\prime}\right)$ for $P E>20.0 \mathrm{MeV}$ were set to 1.0 , slopes of model parameters were set to 0.0 , and neither was varied because preliminary calculations showed that these parameters could not be determined from available data and were assumed not to contribute.

\section{Region Boundries}

The values determined for $\Delta Z\left(A^{\prime}\right), \sigma_{Z}\left(A^{\prime}\right), F_{Z}\left(A^{\prime}\right)$, and $F_{N}\left(A^{\prime}\right)$ from lowenergy data depend on $A^{\prime}$ and can be represented by simple functions in each of several regions of $A^{\prime}$. The region boundries, shown as short dotted lines and labeled B1-6 and Ba,b in Figs. 18a-d, 19a-d, and 20a-d, pp. 31-33, are defined below. The parameters in the functions for each region were calculated by the method of least squares, if there were sufficient data, or, if not, were estimated from derived systematics by using Eq. 17, Table 2, p. 29, with the then current model parameter values. Several cycles of calculation were sometimes necessary. For fission reactions with $P E>\sim 20 \mathrm{MeV}$ only average parameter functions and parameter values could be determined for each reaction (Table 3, p. 30, Figs. 21a-d, p. 34).

$$
\begin{aligned}
& \mathrm{B} 1=70 \\
& \mathrm{~B} 2=77+0.036\left(A_{F}-236\right) \\
& \mathrm{B} 3=A_{F}-\mathrm{B} 4 \\
& \mathrm{~B} 4=\frac{\Delta Z_{\max }-\Delta Z(140)+A_{\max }^{\prime}[S L 50]+140\left[\partial \Delta Z / \partial A^{\prime}\right]}{S L 50+\partial \Delta Z / \partial A^{\prime}} \\
& \mathrm{B} 5=A_{F}-\mathrm{B} 2 \\
& \mathrm{~B} 6=A_{F}-\mathrm{B} 1 \\
& \mathrm{Ba}=A_{\text {max }}^{\prime} \\
& \mathrm{Bb}=A_{F}-A_{\max }^{\prime}
\end{aligned}
$$


Peak regions (B2-B3, B4-B5):

$$
\begin{aligned}
& \Delta Z\left(A_{H}^{\prime}\right)=\Delta Z(140)+\partial \Delta Z / \partial A^{\prime}\left[A_{H}^{\prime}-140\right] \\
& \sigma_{Z}\left(A_{H}^{\prime}\right)=\sigma_{Z}(140)+\partial \sigma_{Z} / \partial\left[A_{H}^{\prime}-140\right] \\
& \sigma_{Z}\left(A_{L}^{\prime}\right)=\sigma_{Z}\left(A_{H c}^{\prime}\right), \quad\left(A_{H c}^{\prime}=A_{F}-A_{L}^{\prime}\right) \\
& F_{Z}\left(A^{\prime}\right)=F_{Z}(140) \\
& F_{N}\left(A^{\prime}\right)=F_{N}(140)
\end{aligned}
$$

Near symmetry region (B3-B4):

$$
\begin{aligned}
& F(A)=1.00 \\
& \mathrm{~B} 3-\mathrm{Ba}: \Delta Z\left(A^{\prime}\right)=\Delta Z(\mathrm{~B} 3)-S L 50\left[A^{\prime}-\mathrm{B} 3\right] \\
& \sigma_{Z}\left(A^{\prime}\right)=\sigma_{50} \\
& \mathrm{Ba}-\mathrm{Bb}: \Delta Z\left(A^{\prime}\right)=\Delta Z(\mathrm{Ba})+A^{\prime}-\mathrm{Ba}[\Delta Z(\mathrm{Bb})-\Delta Z(\mathrm{Ba})] /[\mathrm{Ba}-\mathrm{Bb}] \\
& \sigma_{Z}\left(A^{\prime}\right)=\sigma_{Z}(140)-\left(\partial \sigma_{Z} / \partial A^{\prime}\right)[140-\mathrm{Bb}] \\
& \mathrm{Bb}-\mathrm{B} 4: \Delta Z\left(A^{\prime}\right)=\Delta Z(\mathrm{~B} 4)+S L 50\left[\mathrm{~B} 4-A^{\prime}\right] \\
& \sigma_{Z}\left(A^{\prime}\right)=\sigma_{50} \\
& \quad A_{\max }^{\prime}=F_{1}\left(A K_{1}\right)+F_{2}\left(A K_{2}\right) \\
& \left.\quad F_{1}=\left(250 .-A_{F}\right) / 14 .\right] \quad(\text { Limits } 0.0 \text { and } 1.0) \\
& \quad F_{2}=1.0-F_{1} \\
& A K_{1}=50.0\left(A_{F} / Z_{F}\right)-\delta Z_{\max } / S L 50 \\
& A K_{2}=\left(50.0-\delta Z_{\max }\right)\left(A_{F} / Z_{F}\right)
\end{aligned}
$$


Wing regions (B1-B2, B5-B6):

$$
\begin{aligned}
\Delta Z\left(A_{L}^{\prime}\right) & =\Delta Z(\mathrm{~B} 2)+\Delta Z S L W\left[\mathrm{~B} 2-A_{L}^{\prime}\right] \\
\Delta Z\left(A_{H}^{\prime}\right) & =\Delta Z(\mathrm{~B} 5)-\Delta Z S L W\left[A_{H}^{\prime}-\mathrm{B} 5\right] \\
\sigma_{Z}\left(A_{L}^{\prime}\right) & =\sigma_{Z}\left(A_{H c}^{\prime}\right),\left(A_{H c}^{\prime}=A_{F}-A_{L}^{\prime}\right) \\
\sigma_{Z}\left(A_{H}^{\prime}\right) & =\sigma_{Z}(\mathrm{~B} 5)+\sigma_{Z} S L W\left[\mathrm{~B} 2-A_{L}^{\prime}\right] \\
F_{Z}\left(A_{L}^{\prime}\right) & =F_{Z}(140)+F_{Z} S L W\left[B 2-A_{L}^{\prime}\right] \\
F_{Z}\left(A_{H}^{\prime}\right) & =F_{Z}(140)+F_{Z} S L W\left[A_{H}^{\prime}-\mathrm{B} 5\right] \\
F_{N}\left(A_{L}^{\prime}\right) & =F_{N}(140)+F_{N} S L W\left[\mathrm{~B} 2-A_{L}^{\prime}\right] \\
F_{N}\left(A_{H}^{\prime}\right) & =F_{N}(140)+F_{N} S L W\left[A_{H}^{\prime}-\mathrm{B} 5\right]
\end{aligned}
$$

Far wing regions $(<\mathrm{B} 1,>\mathrm{B} 6)$ :

$$
\begin{aligned}
& \Delta Z\left(A_{L}^{\prime}\right)=\Delta Z(\mathrm{~B} 2) \\
& \Delta Z\left(A_{H}^{\prime}\right)=\Delta Z(\mathrm{~B} 5) \\
& \sigma_{Z}\left(A^{\prime}\right)=\sigma_{Z}(\mathrm{~B} 5) \\
& F_{Z}\left(A^{\prime}\right)=F_{Z}(140) \\
& F_{N}\left(A^{\prime}\right)=F_{N}(140)
\end{aligned}
$$

\section{$Z_{P}$ Near 50}

Available data for low-energy fission reactions show enhanced yields for ${ }_{50} \mathrm{Sn}$ fission products with $A^{\prime}$ just below 130 [WA88]. This effect is represented by the $Z_{P}$ model for U235T by narrow charge dispersion (small $\sigma_{Z}=\sigma_{50}$, (see Fig. $18 \mathrm{a}$, p. 31) and steeply rising $\Delta Z$ near $\Delta\left(Z_{P}=50\right)$ (Fig. 18b, p. 31). The data for the complementary light products from CF249T do not show a decrease in $\sigma_{Z}$ (Fig. 19a, p. 32) but do show an increase in $\Delta Z$ (Fig. 19b, p. 32) that is less steep than $\Delta Z$ for U235T [DA89]. The differences were treated by Eq. 17, Table 2, p. 29, for $\sigma_{50}$ and $S L 50$. The average $\Delta Z_{\max }$ for the two reactions was used for all low-energy fission reactions, but intercepts with $\Delta Z_{P}=50$ were 
different. Data for U235T could best be represented by an intercept at $\Delta Z=0.0$ (Fig.18b), and those for CF249T could best be represented by an intercept at $\Delta Z_{\max }$ (Fig. 19b). A linear function in $A_{F}$ of intercept values from $\Delta Z=0.0$ to $\Delta Z=\Delta Z_{\max }$ was assumed for $A_{F}$ between 236 and 250 . An intercept at $\Delta Z=0.0$ was assumed for $A_{F}<236$, and an intercept at $\Delta Z=\Delta Z_{\max }$ was assumed for $A_{F}>250$.

\section{High-energy Fission}

Data for low-energy fission reactions $(P E \leq \sim 8 \mathrm{MeV})$ and for high-energy fission reactions $(P E>\sim 20 \mathrm{MeV})$ were treated separately, with some data, e.g., from fast and $14-\mathrm{MeV}$ neutron-induced-fission reactions, being used with both data sets to enhance smooth transitions of parameter values from one energy range to the other. The equations and parameter values derived by the method of least squares are shown in Tables 2 and 3, pp. $29 \& 30$. Many parameter values for high-energy fission, Table 3 , are zero or one because parameter slopes and even-odd proton and neutron effects could not be determined from the data available and are assumed to be absent. Thus, for high-energy $(P E>20 \mathrm{MeV})$ $F(A)=1.0, \sigma_{Z}\left(A^{\prime}\right)=\sigma_{Z}(140)$, and $\Delta Z\left(A_{H}^{\prime}\right)=-\Delta Z\left(A_{L}^{\prime}\right)=\Delta Z(140)$. Near symmetry $\Delta Z\left(A_{H}^{\prime}\right)=-\Delta Z\left(A_{L}^{\prime}\right)=0.0$, except the transition to 0.0 near $Z_{P}=50$ is assumed to occur with the same slope $(S L 50)$ as for low-energy fission. (See Table 3 and Figs. 21a-d, p. 34.)

The dependences of $\sigma_{Z}(140)$ and $\Delta Z(140)$ on $P E$ are shown in Figs. 22 and 23, p. 35. The pararameter values derived from data for individual fission reactions are shown as points, and the functions derived (Tables 2 and 3, pp. $29 \& 30)$ from these values are shown as lines. It can be seen that for $\sigma_{Z}((140)$ the transition region (represented by a dotted line) connects the low- and highenergy functions to give an over-all smooth systematic function. For $\Delta \mathrm{Z}(140)$ there are sharp bends in the function, but it is continuous. 


\section{Prefission Proton Emission}

It was found for high-excitation energies $(P E>65 \mathrm{MeV})$ that model representation of data could be improved by including a model parameter, $N P E$, for the number of protons lost before fission, thus reducing $Z_{F}$ to $P Z-N P E$.

$$
N P E=0.0078(P E-65) \text { if } P E>65 \mathrm{MeV} ; N P E=0.0 \text { if } P E \leq 65 \mathrm{MeV}
$$

The NPE function and the parameter values from which it was derived are shown as a line and points, respectively, in Fig. 24, p. 35. Values for the filled points for $P E<20$ were not used in the analysis; they are consistant with the function to within the estimated $\pm \sim 0.5$ uncertainty in the function.

\section{Estimation of Model Uncertainties}

It has been helpful in efforts to represent measured fractional independent and cumulative yields by Gaussian functions, and to use these functions to estimate unmeasured yields, and have an estimate of the uncertainity in the functions. For this purpose Eq. 16, proposed in 1992 [WA92], has been used to estimate the percent uncertainity, $P E R$.

$$
P E R=6+6\left(e^{\left(\left|Z-Z_{P}\right| / \sigma_{Z}\right)}\right)
$$

$\begin{array}{ccccc}\text { Examples: } & \mathrm{FI}\left(\sigma_{Z}=0.6\right) & \left|Z-Z_{P}\right| / \sigma_{Z} & P E R & 1+P E R / 100 \\ & 0.60 & 0.0 & 12 . & 1.12 \\ 0.40 & 1.0 & 22.3 & 1.22 \\ 0.12 & 2.0 & 50.3 & 1.50 \\ 0.015 & 3.0 & 127 . & 2.27 \\ & 1.5 \mathrm{e}^{-5} & 5.0 & 896 . & 9.96 \\ & 3.5 \mathrm{e}^{-10} & 7.0 & 6590 . & 66.90\end{array}$

FI $^{*} P E R / 100$ can be used for the smaller $P E R$ as an estimate of the absolute 
uncertainity. Multiplying and dividing FI by $1+P E R / 100$ for the larger $P E R$ avoids negative lower-limit estimates.

\section{Evaluation of Systematic Model Estimates of Yields}

FI and FC yields were calculated using Gaussian parameters derived from the systematic model functions in Tables 2 and 3, pp. $29 \& 30$, and compared to experimental values as ratios of the two for several fission reactions. Some ratios are plotted in Figs. 25-28, p. 36. It can be seen that the ratios cluster about 1.0, as they should, but that a number deviate considerably from 1.0, especially for the lower experimental FI, FC values, those $<0.01$ (open symbols in the figures). The reduced $\chi^{2}$ values for using systematic model functions and experimental values and errors are considerably greater than 1.0, but, if estimated model errors (Eq. 16) are used in place of experimental errors, the reduced $\chi^{2}$ values, shown in parentheses in Figs. 25-28, are close to 1.0.

An alternative way of comparing experimental data with Gaussian curves derived from model systematic functions is shown in Figs. 29, 30a, and 30b, p. 37. However, this approach can be used only for high-energy reactions for which $\sigma_{Z}\left(\mathrm{~A}^{\prime}\right)$ is assumed constant.

Much of the experimental data for proton-induced fission of ${ }^{232} \mathrm{Th}$ is for $\mathrm{Rb}$ and Cs fission products measured mass-spectrometrically [TC72, NP80]. Two problems were encountered with the FI values derived from the data for the higher energies $(>\sim 50-\mathrm{MeV} p)$. (1) Many FI values fall above the curves as shown in Fig 30a, p. 37, contrary to the requirement that the sum of FI at unit intervals of $A^{\prime}$ be 1.0. To compensate, experimental independent yield data (IN, $\%$ ) were normalized so that the sum for each element ( $\mathrm{Rb}$ or $\mathrm{Cs}$ ) equaled the sum from the model. (2) The renormalized FI for Cs isotopes far from stability (large negative $Z-Z_{P}$ ) still fall well above the same curves in Fig. 30b, p. 37 . 
These Cs FI were not used for determination of model parameters.

The normalization procedure allowed conversion of published [NP80] relative indium yields to absolute values. The indium FI values plotted in Fig. 30b, p. 37, are close to the line representing model values and thus support model treatments of $\sigma_{Z}\left(A^{\prime}\right)$ and $\Delta Z\left(A^{\prime}\right)$ near symmetry. 


\section{TABLE 2 - EQUATIONS FOR SYSTEMATIC TRENDS IN Zp PARAMETERS AT LOW ENERGY $(P E \leq 8 \mathrm{MeV})$}

\begin{tabular}{|c|c|c|c|c|c|c|}
\hline Par. & $P(1)$ & $P(2)$ & $P(3)$ & $P(4)$ & $P(5)$ & No. ${ }^{a}$ \\
\hline
\end{tabular}

Parameters for regions near peaks (B2-3, B4-5)

\begin{tabular}{lcccccc}
$\sigma_{Z}(140)$ & 0.566 & 0.0 & 0.0064 & 0.0109 & 0.0 & 13 \\
$\Delta_{Z}(140)$ & -0.487 & 0.0 & 0.0180 & 0.0 & -0.00203 & 13 \\
$F_{Z}(140)$ & 1.207 & 0.0 & -0.0420 & 0.0 & 0.0022 & 13 \\
$F_{N}(140)$ & 1.076 & 0.0 & 0.0 & 0.0 & 0.0 & 12 \\
$\sigma_{Z} S L$ & -0.0038 & 0.0 & 0.0 & 0.0 & 0.0 & 6 \\
$\Delta_{Z} S L$ & -0.0080 & 0.0 & 0.0 & 0.0 & 0.0 & 6 \\
$F_{Z} S L^{b}$ & 0.0030 & 0.0 & 0.0 & 0.0 & 0.0 & 6 \\
\hline
\end{tabular}

Parameters for regions near symmetry (B3-4)

\begin{tabular}{lcccccc}
\hline & & & & & & \\
$S L 50$ & 0.191 & 0.0 & -0.0076 & 0.0 & 0.0 & 4 \\
$\sigma_{Z} 50$ & 0.356 & 0.060 & 0.0 & 0.0 & 0.0 & 4 \\
$\Delta_{Z} \max$ & 0.699 & 0.0 & 0.0 & 0.0 & 0.0 & 2 \\
\hline
\end{tabular}

Parameters for wing regions $(\mathrm{B} 1-2, \mathrm{~B} 5-6)^{c}$

\begin{tabular}{ccccccc}
$\sigma_{Z} S L W$ & -0.045 & 0.0094 & 0.0 & 0.0 & 0.0 & 3 \\
$\Delta_{Z} S L W$ & 0.0 & -0.0045 & 0.0 & 0.0 & 0.0 & 3 \\
$F_{Z} S L W$ & 0.159 & -0.028 & 0.0 & 0.0 & 0.0 & 3 \\
$F_{N} S L W$ & 0.039 & 0.0 & 0.0 & 0.0 & 0.0 & 3 \\
\hline
\end{tabular}

${ }^{a}$ Number of parameter values used as data in least-squares calculation.

${ }^{b}$ The weighted averages for the $\mathrm{F}_{Z}$ and $\mathrm{F}_{N}$ slopes from 6 values for each are: 0.0030(7) and -0.0006(10), with reduced-chi-square values of 0.85 and 1.59 , respectively. For odd- $Z$ fissioning nuclides the $\mathrm{F}_{Z}$ for light products is taken to be 1.032(10), the average of 2 values, and the recriprocal of this value is used for heavy products. (See discussion.)

${ }^{c}$ Wing functions with large slopes are assumed to start at:

$$
A_{L}^{\prime}=77.0+0.036\left(A_{F}-236\right) \text { and } A_{H}^{\prime}=A_{F}-A_{L}^{\prime}
$$

and at the value of each parameter for the peak regions at these $A^{\prime}$ values. The function was derived empirically by trial and error. Values of $A_{L}^{\prime}$ for U235T, PU239T, and CF249T are, respectively, 77.0, 77.1, and 77.5. The wing slopes for $A_{H}^{\prime}$ are the same as for the $\sigma, F_{Z}$, and $F_{N}$ for complementary $A_{L}^{\prime}$; for $\Delta$ the magnitude of the correction is the same but opposite in sign to maintain charge conservation. 
TABLE 3 - EQUATIONS FOR SYSTEMATIC TRENDS IN Zp MODEL PARAMETERS AT HIGH ENERGY $(P E \leq 20 \mathrm{MeV})$

$$
\begin{aligned}
& \begin{array}{c}
P A R=P 1+(P 2-P 1)\left(1.0-e^{-P(5)(P E)}\right) \\
P 1=P(1)+P(3)\left[Z_{F}-92\right] \\
P 2=P(2)+P(4)\left[Z_{F}-92\right]
\end{array} \\
& \begin{array}{lllllll}
\text { Par. } & P(1) & P(2) & P(3) & P(4) & P(5) & \text { No. }^{a} \\
\hline
\end{array} \\
& \text { Parameters for regions near peaks (B2-3, B4-5) } \\
& \begin{array}{lllllll}
\sigma_{Z} & 0.542 & 1.310 & 0.033 & 0.0 & -0.005 & 17
\end{array} \\
& \begin{array}{lllllll}
\Delta_{Z} & -0.428 & 0.0 & 0.0 & 0.164 & -0.0116 & 17
\end{array} \\
& \begin{array}{llllll}
F_{Z}(140) & 1.0 & 0.0 & 0.0 & 0.0 & 0.0
\end{array} \\
& \begin{array}{llllll}
F_{N}(140) & 1.0 & 0.0 & 0.0 & 0.0 & 0.0
\end{array} \\
& \begin{array}{llllll}
\sigma_{Z} S L & 0.0 & 0.0 & 0.0 & 0.0 & 0.0
\end{array} \\
& \begin{array}{llllll}
\Delta_{Z} S L & 0.0 & 0.0 & 0.0 & 0.0 & 0.0
\end{array} \\
& \begin{array}{llllll}
F_{Z} S L & 0.0 & 0.0 & 0.0 & 0.0 & 0.0 \\
\hline
\end{array} \\
& \text { Parameters for regions near symmery (B3-4) } \\
& N P E=0.0078(P E-65) \text { if } P E>65-N P E=0.0 \text { if } P E \leq 65
\end{aligned}
$$

\begin{tabular}{lcccccc}
$S L 50^{b}$ & 0.191 & 0.0 & -0.0076 & 0.0 & 0.0 & 4 \\
$\sigma_{Z} 50^{c}$ & 0.542 & 1.310 & 0.033 & 0.0 & -0.005 & 17 \\
$\Delta_{Z} \max$ & 0.0 & 0.0 & 0.0 & 0.0 & 0.0 & \\
\hline
\end{tabular}

\begin{tabular}{llllll}
$\sigma_{Z} S L W$ & 0.0 & 0.0 & 0.0 & 0.0 & 0.0 \\
$\Delta_{Z} S L W$ & 0.0 & 0.0 & 0.0 & 0.0 & 0.0 \\
$F_{Z} S L W$ & 0.0 & 0.0 & 0.0 & 0.0 & 0.0 \\
$F_{N} S L W$ & 0.0 & 0.0 & 0.0 & 0.0 & 0.0 \\
\hline
\end{tabular}



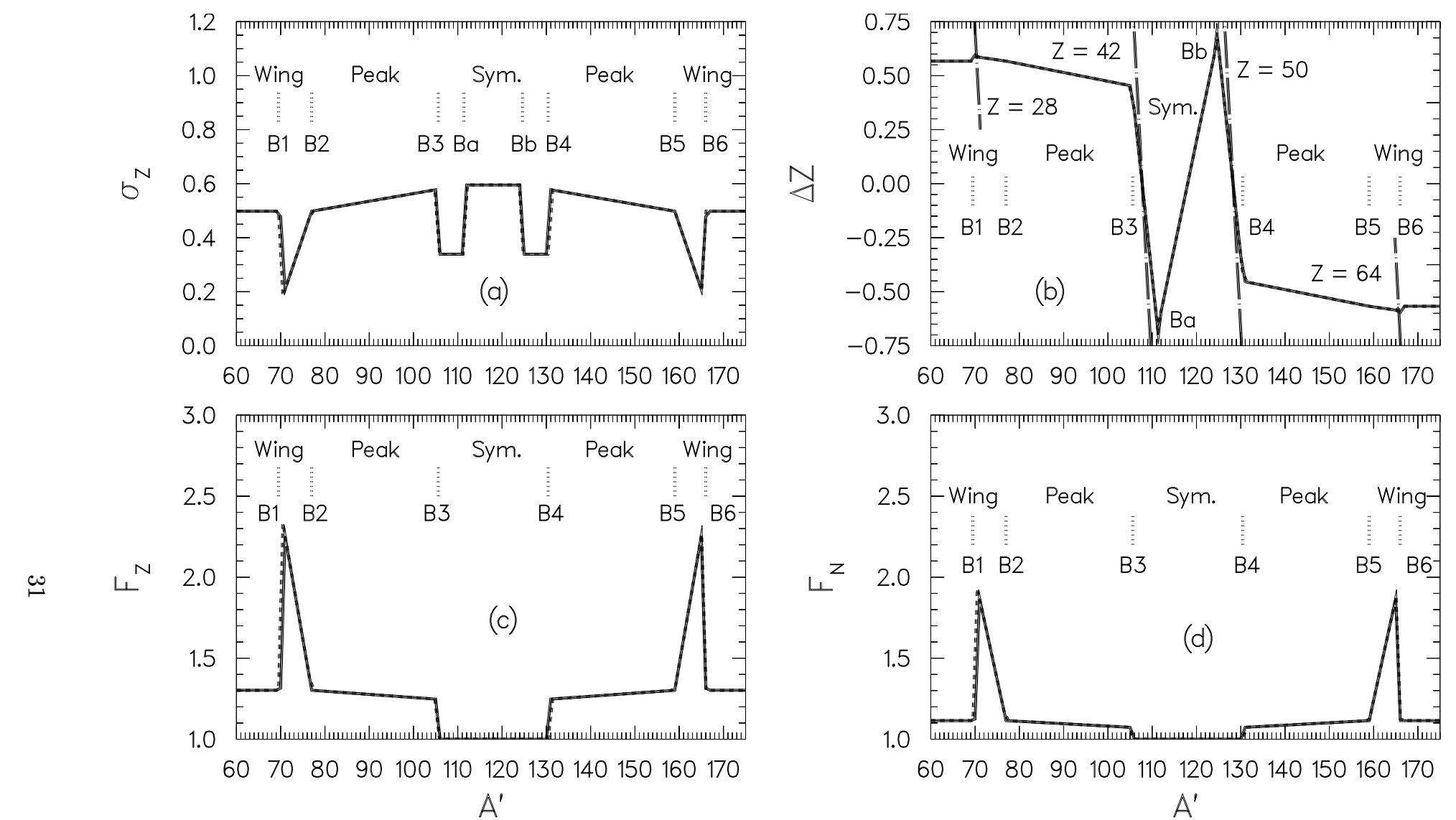

60708090100110120130140150160170

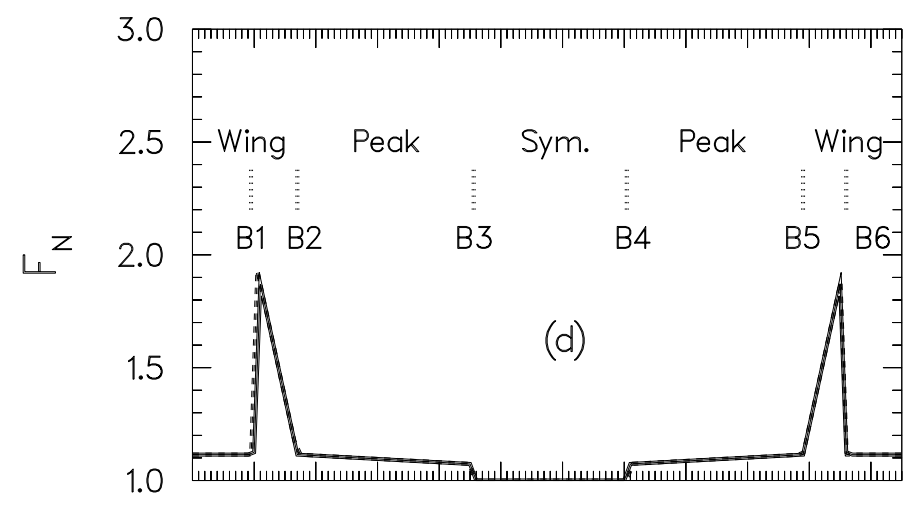

$6070 \quad 8090100110120130140150160170$

Fig. $18-Z_{P}$ Functions for U235T

Solid lines from CYF systematics, red. $\chi^{2}=7.9(0.8)$

Dashed lines calculated by L.S., red. $\chi^{2}=2.9(0.8)$ 

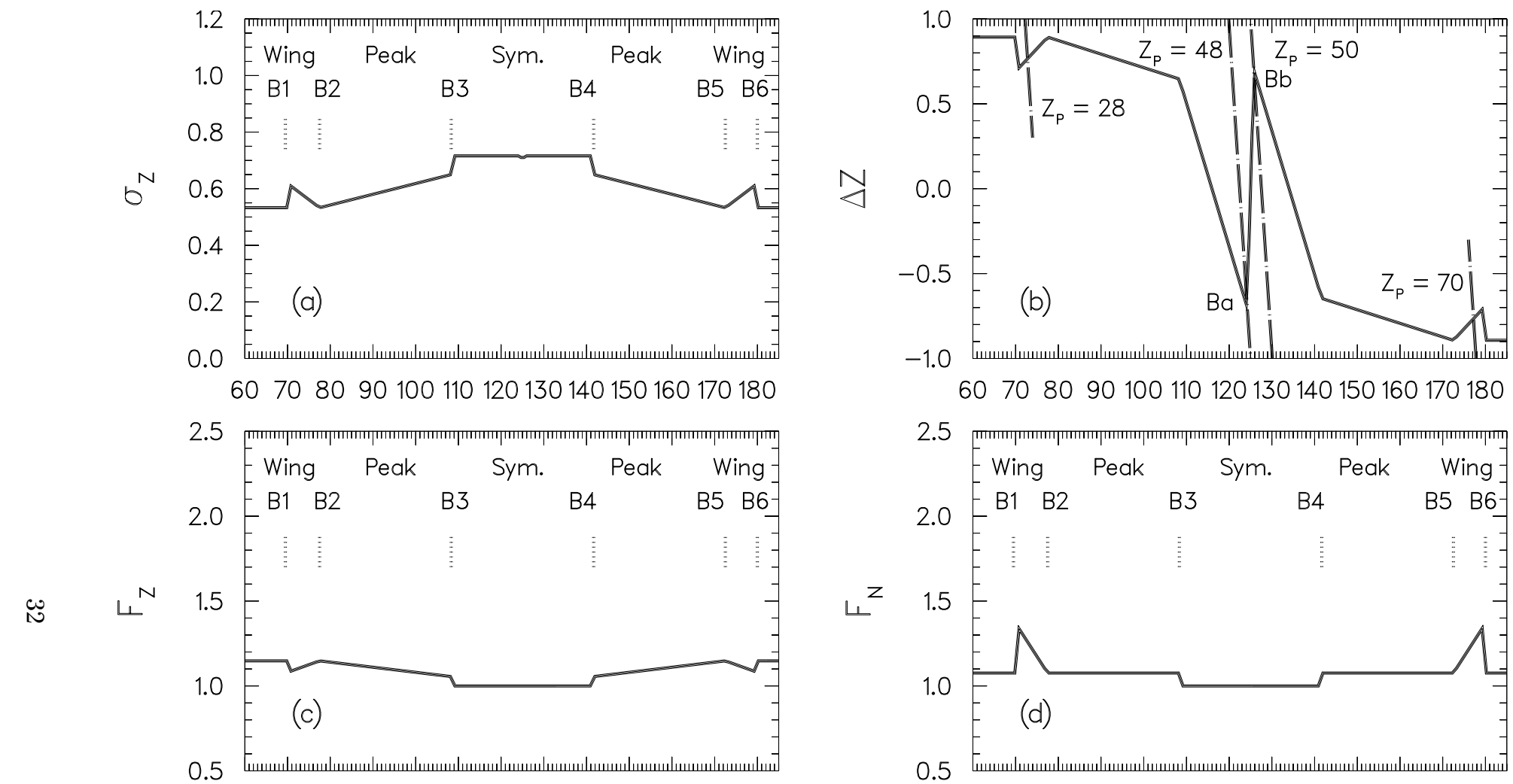

60708090100110120130140150160170180 $A^{\prime}$

60708090100110120130140150160170180 $A^{\prime}$

Fig. 19 -Systematic $Z_{P}$ Parameter Functions for CF249T, red. $\chi^{2}=2.9(1.0)$ 

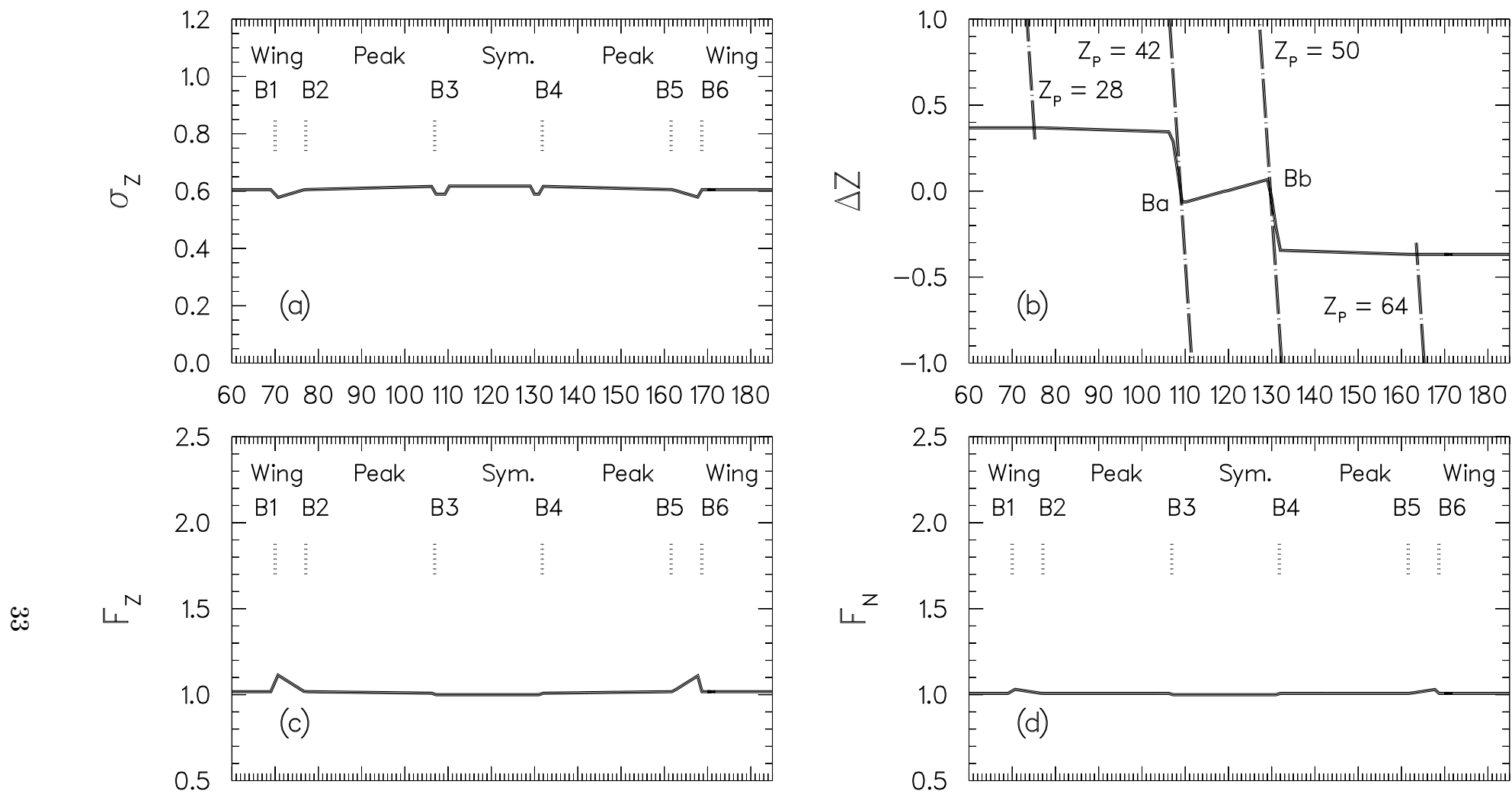

60708090100110120130140150160170180 $A^{\prime}$

60708090100110120130140150160170180 $A^{\prime}$

Fig. 20 -Systematic $\mathrm{Z}_{P}$ Parameter Functions for U238H, red. $\chi^{2}=7.2(1.2)$ 

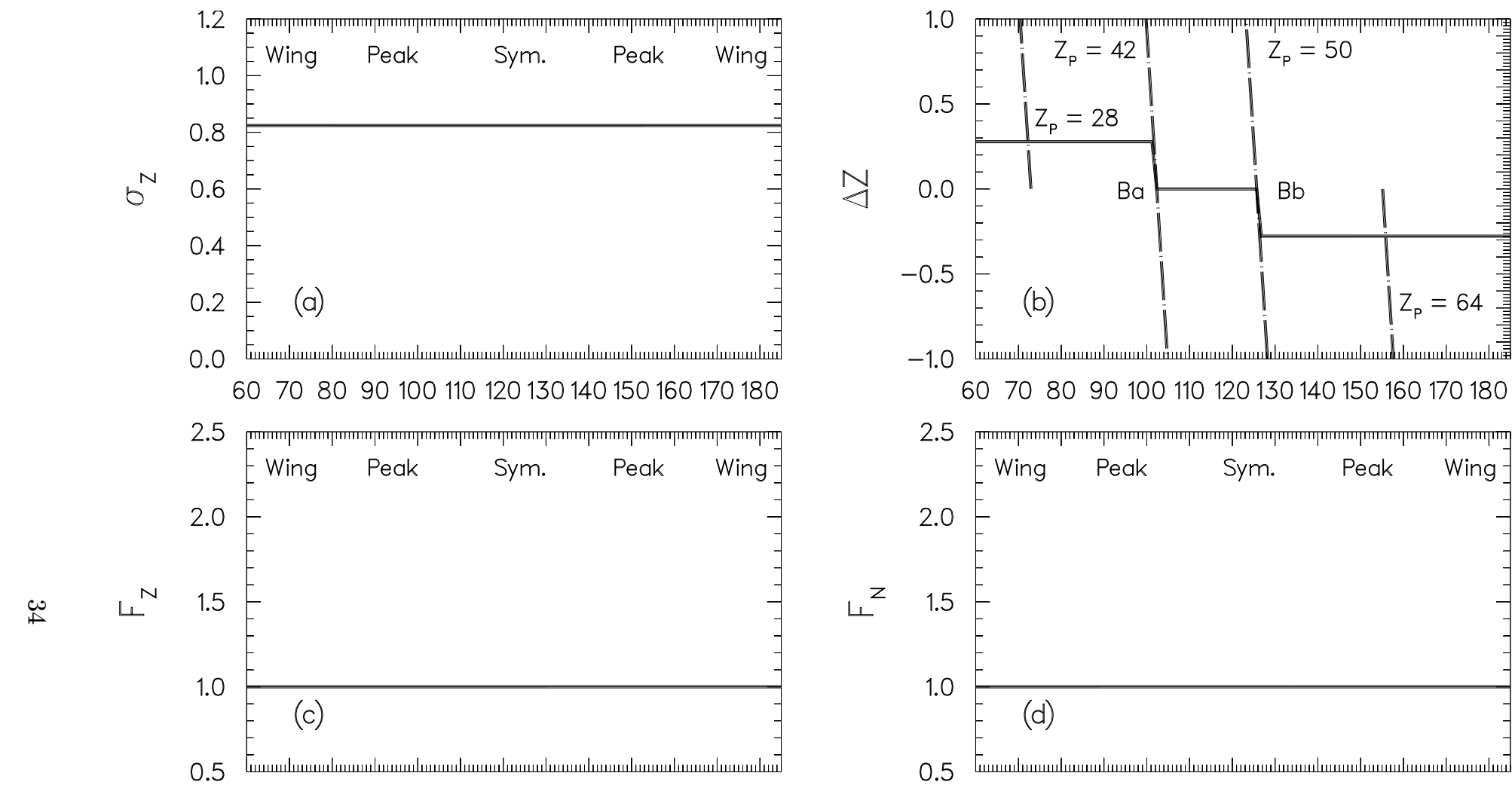

60708090100110120130140150160170180 $A^{\prime}$ 


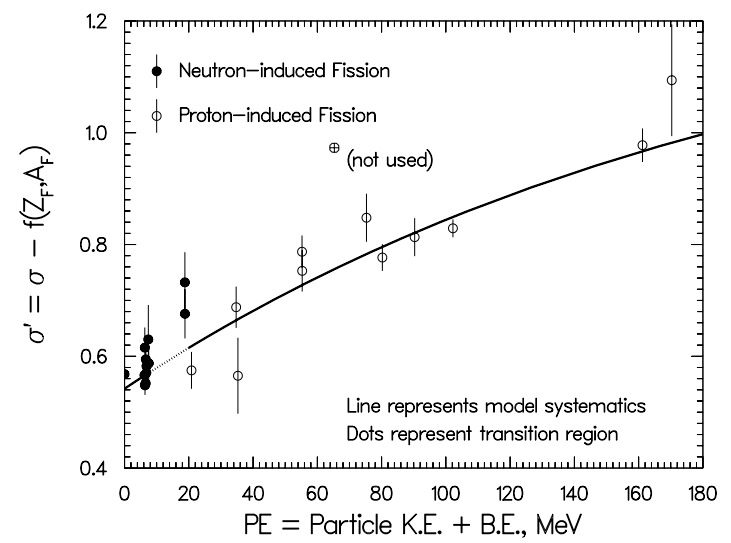

Fig. 22 - Nuclear Charge Distribution Width Parameter, $\sigma$

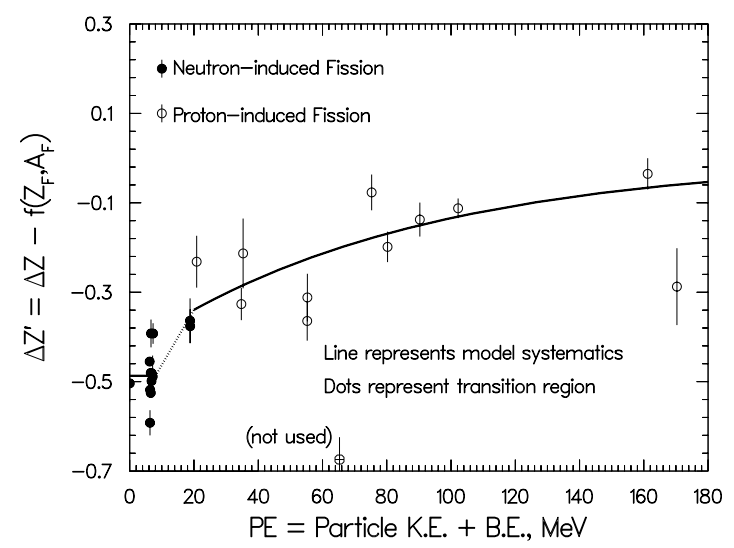

Fig. 23 - Nuclear Charge Displacement Parameter, $\Delta \mathrm{Z}$

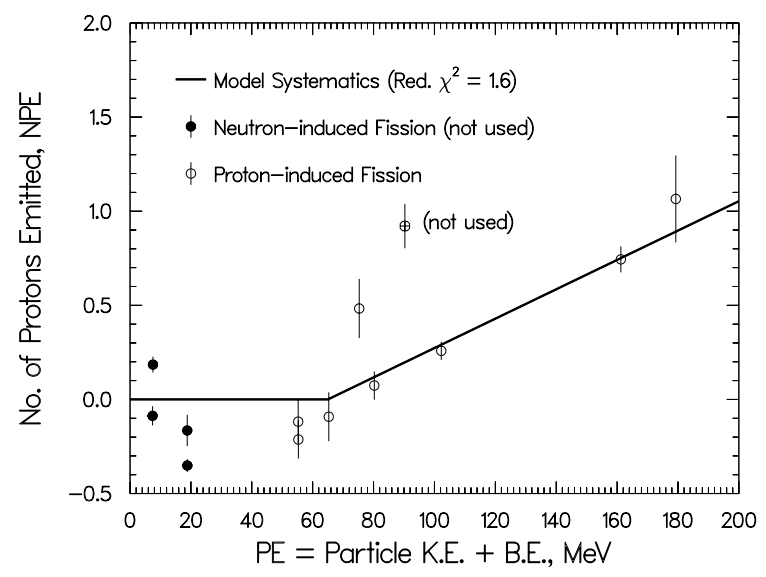

Fig. 24 - Number of Protons Emitted before Fission 


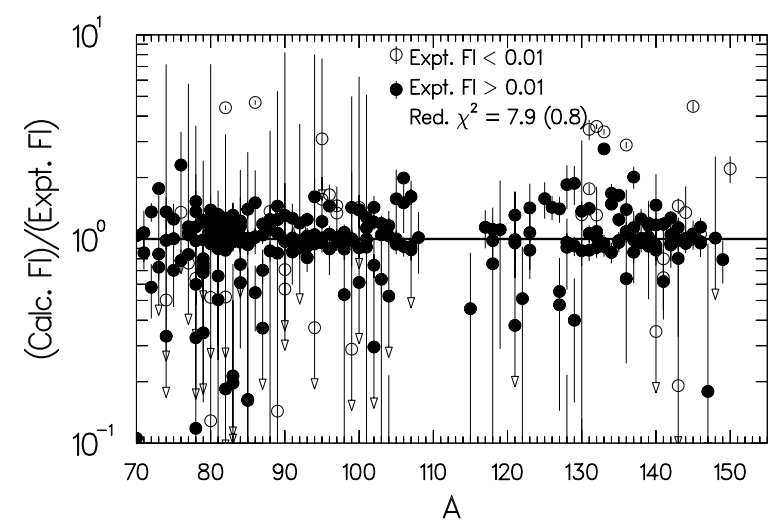

Fig. 25 - Ratios of Sys. Fl to Expt. Fl for U235T

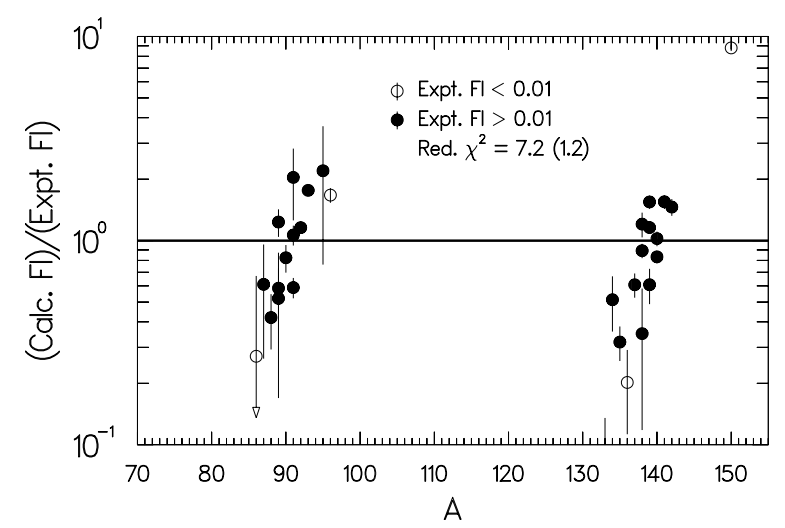

Fig. 27 - Ratios of Sys. Fl to Expt. Fl for U238H

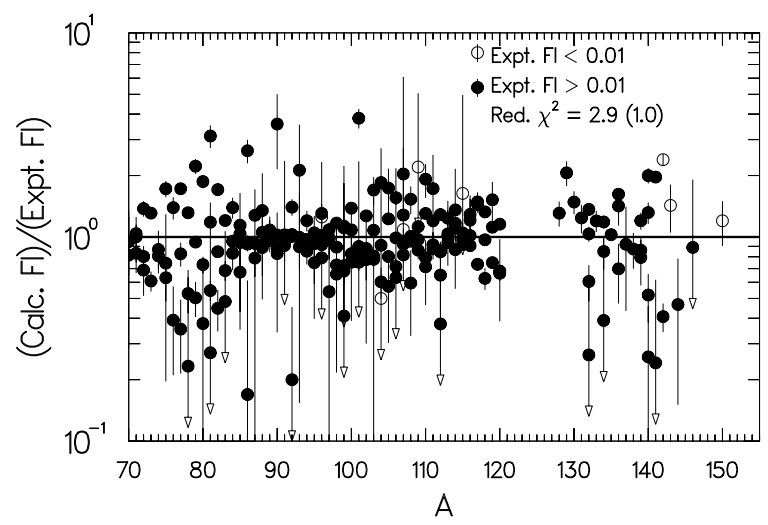

Fig. 26 -Ratios of Sys. Fl to Expt. Fl for CF249T

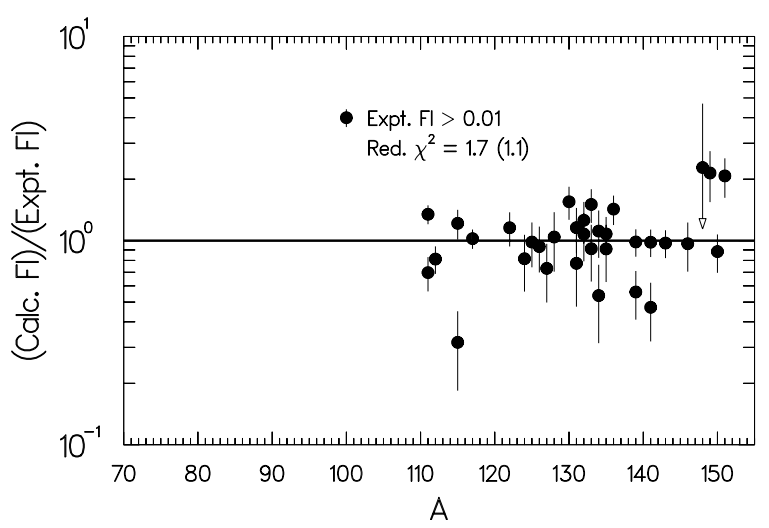

Fig. 28 -Ratios of Sys. Fl to Expt. Fl for ${ }^{238} \mathrm{U}+85-\mathrm{MeV}$ p 


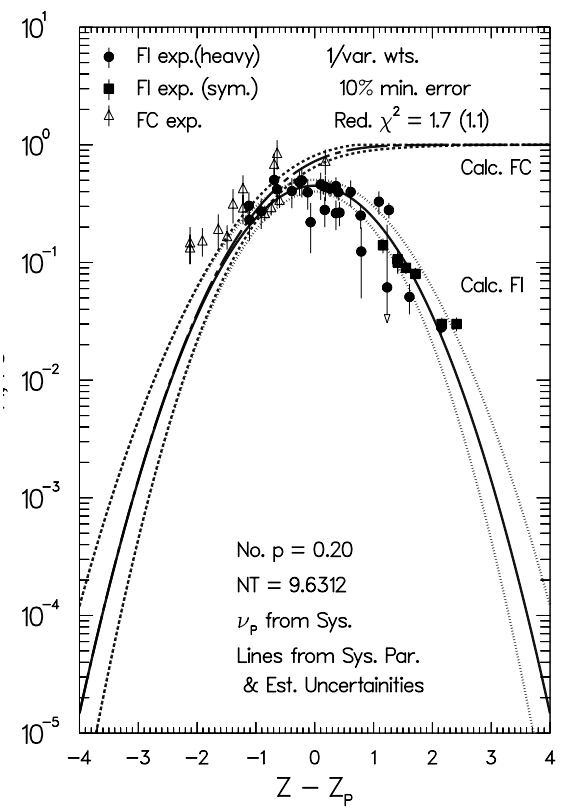

Fig. 29 - Exp. Data \& Sys. Curves for ${ }^{238} \mathrm{U}+85-\mathrm{MeV}$ p

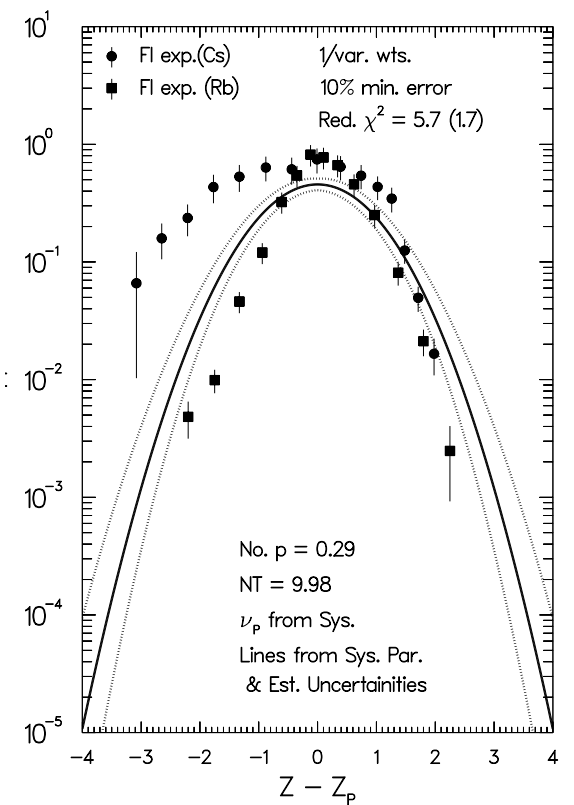

Fig. 30a - Exp. MS \& Sys. Curves for ${ }^{232} \mathrm{Th}+97-\mathrm{MeV} \mathrm{p}$

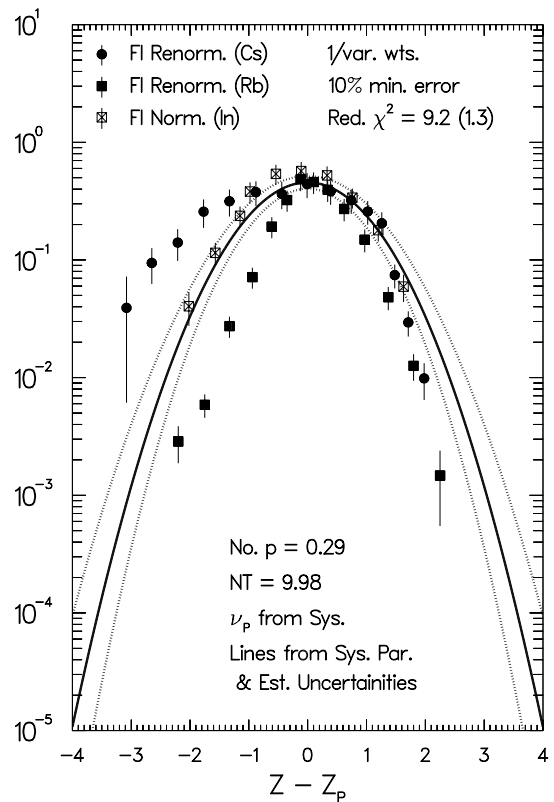

Fig. 30b-Renorm. MS \& Sys. Curves for ${ }^{232} \mathrm{Th}+97-\mathrm{MeV} \mathrm{p}$ 


\section{MODELS FOR NEUTRON EMISSION}

The average total number, $N T$, of nucleons emitted before and after scission and the average number of neutrons, $\nu_{A}(A)$, emitted promptly after scission to form products with mass number $\mathrm{A}$ are used in model calculations of mass and nuclear-charge distributions, respectively, as has been described in the preceeding two sections. To review, NT is used to determine the central mass number, $A_{c}=(P A-N T) / 2$, in multi-Gaussian mass-yield calculations. The quantity $\nu_{A}(A)$ is used to approximate complementarity in $\mathrm{A}$ of light- and heavy-fission products, $A_{L}^{\prime}+A_{H}^{\prime}=A_{F}, A^{\prime}=A+\nu_{A}(A), \nu_{A}(A)=\nu_{T}(A) R(A), \nu_{T}(A)$ being the average number of neutrons emitted to form products with mass number A and their complements, and $R(A)$ being the fraction of these neutrons that from products with A [WA88, WA99].

In the earlier models [WA88, WA89, WA92, WA99] a file of $\nu_{A}(A)$ values was required for each fission reaction treated. Below, a model is proposed that allows calculation of $\nu_{A}(A)$ and, therefore, FI, etc. for any fission reaction in the mass, charge, and energy regions investigated.

\section{Low Energy Systematics}

For $P E<\sim 8 \mathrm{MeV}\left(E_{N}<\sim 2 \mathrm{MeV}\right)$ there are no, or very few, pre-fission neutrons, so $N T=\nu_{F}$ - the number of prompt neutrons emitted following scission - is a good approximation. The quantity $\nu_{F}$ is often called $\bar{\nu}$, although sometimes in the litature $\bar{\nu}$ refers to $N T$.

The quantity $N T$ has been determined experimentally for a number of fission reactions with $P E<\sim 20 \mathrm{MeV}$ [MK72, MI95, JM91, ER93, HO77, AX84, DW86]. Estimates of unmeasured or model $N T$ values can be made from Howerton's equation 8 [HO77], or, as is done here, from a modified equation 8 of 
the same form but with term coefficients re-evaluated by the method of leastsquares. Several hundred $(\sim 1 / 3)$ of the $N T$ values, including values for fission reactions with neutron energies up to $\sim 14 \mathrm{MeV}$, were selected from the extensive compilation of Manero and Konshin [MK72]. Use of experimental NT values from the higher-energy neutron-induced reactions causes $N T$ values calculated from Eq. 21 to include pre-fission neutrons, which can amount to about one per fission for $14-\mathrm{MeV}$ neutron-induced fission reactions. (See later in this section.) The last term in Eq. 21 is omitted for spontaneous fission.

$$
\begin{gathered}
N T=2.286+0.147\left(Z_{F}-92\right)+0.054\left(A_{F}-236\right)+0.040\left(2-F_{Z}-F_{N}\right) \\
+\left(0.145-0.0043\left[A_{F}-236\right]\right)\left(E_{N}-T H\right) \\
F_{Z}=[-1]^{Z_{F}}, F_{N}=[-1]^{\left(A_{F}-Z_{F}\right)} \\
T H=11.47-0.166\left(Z_{F}\right)^{2} / A_{F}+0.093\left(2-F_{Z}-F_{N}\right)-B N
\end{gathered}
$$

The symbols $B N, T H$, and $E_{N}$ represent the binding energy, the threshold, and the particle kinetic energy, respectively.

The average number $\left(\nu_{F}\right)$ of prompt neutrons emitted post fission and used in the derivation of $\nu_{A}(A)$ functions can be obtained by subtracting the average numbers of pre-fission neutrons and beta-delayed neutrons from NT. For lowenergy fission reactions with excitation energies $<\sim 8 \mathrm{MeV}$, pre-fission neutrons have been neglected. The number of beta-delayed neutrons, $\nu_{D}$, is small, about one percent or less of $N T$, and has been neglected.

\section{Modified Terrell Method}

The total number of neutrons emitted to form complementary fission products, $\nu_{T}(A)$, has been derived [WA88, WA89, WA92, WA99] from mass distributions of fission products by a modification [WA88] of Terrell's summation method 
[TE62]. The mass-yield values used recently [WA99] are arithmetic-average values of evaluated averages of experimental data from ENDF/B-VI [ER93], from UKFY-3 [MI95], and from recent data [DE95, SA89, HE94, DI91, ST96, MS96] not included in the evaluated average values. Model $Y(A)$ values [WA99] were used where there were no experimental data. A value of $\nu_{T}(A$ at symmetry must be assumed for the calculation, and a value of 4.0, or $(N T+1.0)$, if larger was used. For the fissioning nuclei treated, $Z_{F}=90-98$, a $\nu_{T}(A)$ value near symmetry appreciably larger than $N T$ is consistent with the observed low fissionfragment kinetic energies near symmetry [BB83] that suggest an elongated scission configuration resulting in highly excited fission fragments, which emit more than the average number of neutrons following scission.

The modified Terrell summation method is illustrated in Fig. 31, p. 46, where the sums of light and of heavy mass-number yields, starting at the wings, are plotted (points) along with smooth functions (solid lines) fitted to the points. Values of $\nu_{T}(A)$ are taken to be the absolute differences between point values and complementary function values, illustrated in Fig. 31 by horizontal dotted lines for $A=95$ and for $A=144$. In earlier treatments [WA88, WA89, WA92, WA99], the $\nu_{T}(A)$ values obtained (circles in Figs. 32-37, pp. $46 \& 47$ ) were used with an $R(A)$ function for each fission reaction to calculate sets of $\nu_{A}(A)$ values that were smoothed and normalized. Below, empirical models are proposed for $\nu_{T}(A)$ and $R(A)$.

\section{Trends in $\nu_{T}(A)$}

The derived values of $\nu_{T}(A)$, shown as circles in Figs. 32-37, pp. $46 \& 47$, are approximately constant for each of a number of fission reactions, except for some structure near symmetry and for very asymmetric mass division. In addition to the peak at symmetry, a result of the assumption of four or more neutrons being emitted near symmetry, there are small dips on either side of the peak, as 
can be seen from the circles in Figs. 32 to 37. The dips occur near $A_{H}=130$, and in the complementary light-product range, near $A=104$ for U235T, and may be associated with condensed scission configurations, possibly related to the proximity of $Z$ and $N$ of the heavy fragment to the spherical $Z=50$ and $N=82$ shells. High fragment kinetic energies should result, and are observed [BB83], and, therefore, excitation energy and neutron emission are small.

The dips in the $\nu_{T}(A)$ function are present for the fission reactions of uranium, plutonium, and americium isotopes that were investigated, but are smaller or absent for the fission reactions investigated of thorium and californium isotopes. This may be due, at least in part, to the somewhat fewer experimental mass-yield values for these reactions, since, as shown in Fig. 38, p. 47, dips are absent when model mass-yields, calculated from smooth functions, are used for U235T.

Other structure appears for very asymmetric mass division (see Fig. 32-37, pp. $46 \& 47$ ), but the effects vary in position, magnitude, and sign for the various fission reactions investigated and are attributed to the method as applied to low and, possibly, uncertain fission yield values that form the wings of mass-yield curves.

\section{Model for $\nu_{T}(\mathbf{A})$}

The $\nu_{T}(A)$ values from the $Y(A)$ summation calculations, circles in Figs. 32 to 37 , pp. $46 \& 47$, can be represented, approximately, the solid lines in these figures, by adding three Gaussian functions to a constant, NT, Eq. 22. The $E C O R$ term serves to damp out the peak and dips at high energies $(P E)$, where single-particle effects are less important.

$$
\nu_{T}(A)=N T+E C O R\left[\nu_{S}(A)+\nu_{H}(A)+\nu_{L}(A)\right]
$$




$$
E C O R=e^{-0.05 P E}
$$

For the peak at symmetry:

$$
\begin{gathered}
\nu_{S}(A)=I_{P}\left[e^{\left(A-S_{P}\right)^{2} / C_{P}}\right] / \sqrt{\pi C_{P}} \\
I_{P}=20.0,=0.0 \text { for S.F. } \\
C_{P}=2.0\left(6.0^{2}+1 / 12\right)=72.17 \\
S_{P}=\left(A_{F}-\nu_{S}\right) / 2.0, \nu_{S}=4.0 \text { or }(N T+1.0) \text { if }>4.0
\end{gathered}
$$

For the $\operatorname{dip}$ at $A=130$ :

$$
\begin{gathered}
\nu_{H}(A)=I_{D}\left[e^{-(A-130)^{2} / C_{D}}\right] / \sqrt{\pi C_{D}} \\
I_{D}=-5.0 \\
C_{D}=2.0\left(3.0^{2}+1 / 12\right)=18.17
\end{gathered}
$$

For the light dip complementary to $A=130$ :

$$
\begin{gathered}
\nu_{L}(A)=I_{D}\left[e^{-\left(A-S_{C}\right)^{2} / C_{D}}\right] / \sqrt{\pi C_{D}} \\
S_{C}=A_{F}-130.0-\left(\nu_{F}-D_{H}+S_{H}\right) \\
D_{H}=I_{D} / \sqrt{\pi C_{D}} \\
S_{H}=I_{D}\left[e^{\left.-(130.0-S Y M)^{2} / C_{D}\right)}\right] / \sqrt{\pi C_{D}} \\
S Y M=\left(A_{F}-\nu_{S}\right) / 2.0
\end{gathered}
$$

Model for $R(A)$

Generalized $R(A)$ functions consist of a number of straight-line segments chosen to give saw-tooth $\nu_{A}(A)$ functions, with some inflections. Two $R(A)$ functions are shown in Figs. 39 and 40, p. 47, and equations for the segments in the various regions, designated in Figs. 39 and 40, are given below. Note 
that Eq. 23c and Eq. 23f are excitation-energy dependent continuous functions, which approximate the former discrete values [WA88, WA99].

$$
\begin{aligned}
& A<D \text { : } \\
& R(A)=0.20 \\
& A=D \text { to } F: \\
& R(A)=0.2+(A-D)(R(F)-0.2) /(F-D) \\
& A=F \text { to } G \text { : } \\
& R(A)=0.9-0.0075(P E) \\
& A=G \text { to } H: \quad R(A)=0.5+(H-A)(R(G)-0.5) /(H-G) \\
& A=H \text { to } J \text { : } \\
& R(A)=0.5-(A-H)(0.5-R(J)) /(J-H) \\
& A=J \text { to } K: \\
& R(A)=0.1+0.0075(P E) \\
& A=K \text { to } M \text { : } \\
& R(A)=R(K)+(A-K)(0.8-R(K)) /(M-K) \\
& A>M \text { : } \\
& R(A)=0.80
\end{aligned}
$$

The $\nu_{A}(A)$ functions derived from the above equations for two fission reactions are compared in Figs. 41 and 42, p. 48, to experimental data (U235T: [MR67, BM71, AG65, MN84]; CF252S: [SB73, WB77, GI79, BM63, SH83]). As can be seen the model $\nu_{A}(A)$ functions represent the data moderately well, about as well as the different data sets for each fission reaction agree with each other. The inflection in the $\nu_{A}(A)$ model function near $A=105$, also shown by the data, is related to the the light dip in the $\nu_{T}(A)$ function. The heavy $\nu_{T}(A) \operatorname{dip}$ is reinforced by the $J-K R(A)$ minimum to give the $\nu_{A}(A)$ minimum just below $A=130$. The above equations are used in the current yield-calculation program, CYF.

The details of the $R(A)$ function are believed to be not critical because the complementarity of light and heavy fission products, used in both the $Z_{P}$ and $A_{P}^{\prime}$ nuclear-charge distribution models, is established by $\nu_{T}(A)$, and, although the details of the division of $\nu_{T}(A)$ between light and heavy products may affect values of the model parameters a little, the small changes in parameter values tend to be compensating for yield calculations; differences in calculated yields are very small when different $R(A)$ functions are used. 


\section{High Energy Systematic Trends}

Nucleons may be emitted before fission during de-excitation of highly excited nuclei. At moderate excitation, only a few neutrons are emmitted, and then, if there is sufficient excitation, a nucleus may fission - "second, third, etc. chance fission." This process can be inferred from step-wise increases in fission cross sections with increasing neutron energy about every $6 \mathrm{MeV}$, approximately the neutron binding energy [VH73, HE63]. If it is assumed that the nearly flat cross section between increases can be extrapolated to higher energies, one may estimate from plots of fission cross section vs. neutron energy [BN65] the contributions from "first, second, etc. chance fission." For example, this treatment gives for $14-\mathrm{MeV}$ neutron-induced fission the following average percent contributions from four fission reactions, Th232H, U235H, U238H, and Pu239H: $48 \%$ 1st, $36 \%$ 2nd, $16 \%$ 3rd chance fission.

Thus, the yields of products from $14-\mathrm{MeV}$ neutron induced fission come from three different fissioning nuclei with different excitation energies. Also, the number of neutrons emitted, NT $=\sim 4.0$, are partly pre-scission neutrons. An estimate of the average number of pre-fission neutrons can be made from the percent contributions given above: $0.36+2(0.16)=0.68$. The average number of postfission neutrons, obtained by difference, is $\nu_{F}=\sim 3.3$.

There are few experimental data for post-fission-neutron emission, $\nu_{F}$, for high-energy fission reactions. Only two references were found [CF70, BW64], giving values plotted in Fig. 43, p. 48, as filled squares. Also, the average value of $\sim 3.3$ deduced above for fission by $14-\mathrm{MeV}$ neutrons, is plotted as a filled circle.

Other published values are differences between $N T$ and the number of prefission nucleons calculated by Monte Carlo programs. Values of NT were determined from centroids of relative independent yield distributions of nearly com- 
plementary elements, usually $\mathrm{Rb}$ and $\mathrm{Cs}$, [SY76, DM74, TC72]. The $\nu_{F}$ values, normalized to model $N T$ values, are plotted as open symbols in Fig. 43, p. 48. Fig. 43 also shows the Eq. 24 function as a line that represents, approximately, the various derived values, the points.

$$
\nu_{F}=2.8+3.5\left(1.0-e^{-0.01 P E}\right)
$$

Functions representing ratio values, $R(A)$, were developed to give calculated $\nu_{A}(A)$ values in approximate agreement with experimental $\nu_{A}(A)$ vs. $A$ plots [BW64, CF68, BV70, BF70, CF70]. All plots of experimental data showed that the depth of the dip in $\nu_{A}(A)$ near $A=130$ decreased with increasing energy, but that the low $\nu_{A}(A)$ at low $A$ and the high $\nu_{A}(A)$ at high $A$ changed very little with energy. These trends were represented by replacing equations for $R(A)$ in the $F-G$ and $J-K$ ranges for low energies, Eq. 23c and 23f, by Eq. 25a and $25 \mathrm{~b}$ for high energies.

$$
\begin{array}{ll}
A<F \text { to } G: & R(A)=0.5+0.5 e^{-0.05 P E} \\
A=J \text { to } K: & R(A)=0.5-0.5 e^{-0.05 P E}
\end{array}
$$

The resulting $R(A)$ function multiplied by $\nu_{F}$, Eq. 24, gives $\nu_{A}(A)$ values for the high-energy fission reactions of interest. The results for 30,50 , and 85 $\mathrm{MeV}$ proton induced fission of ${ }^{238} \mathrm{U}$ are shown in Fig. 44, p. 48. Values of $\nu_{A}(A)$ for $\mathrm{U} 238 \mathrm{~F}$ are shown for comparison. 


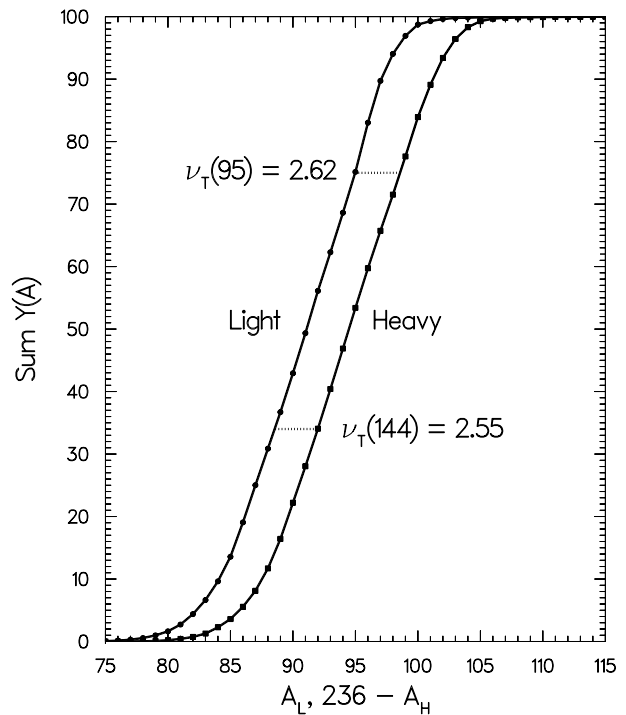

Fig. 31 -Modified Terrell Plot for U235T

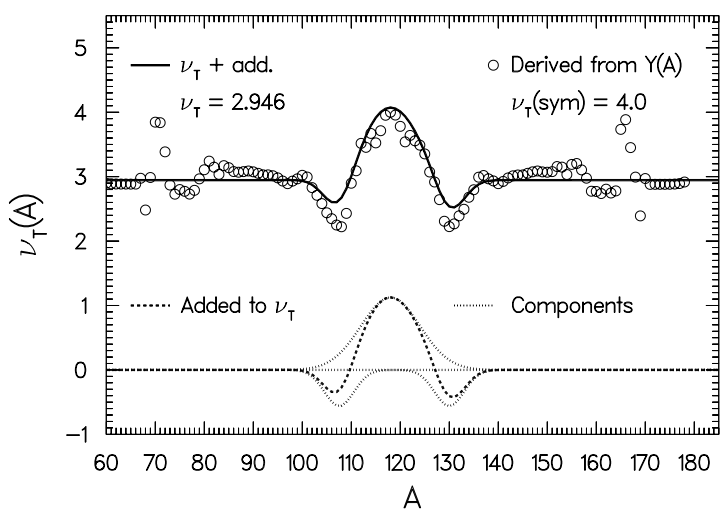

Fig. $33-\nu_{T}$ (A) for PU239T

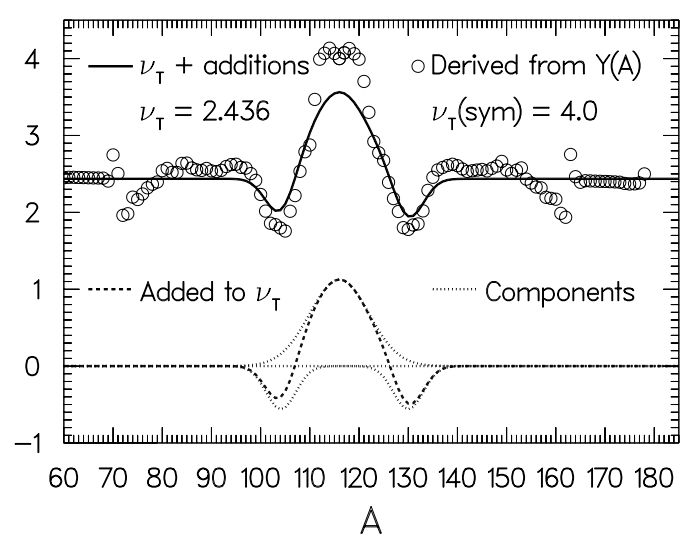

Fig. $32-\nu_{T}(\mathrm{~A})$ for U235T

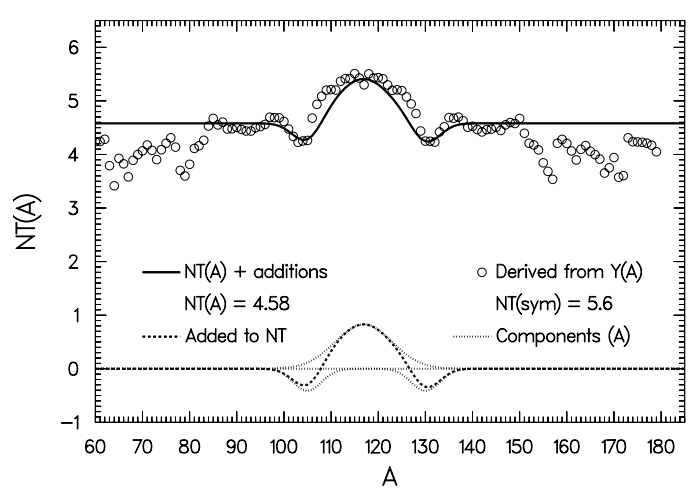

Fig. $34-\mathrm{NT}(\mathrm{A})$ for $\mathrm{U} 238 \mathrm{H}$ 


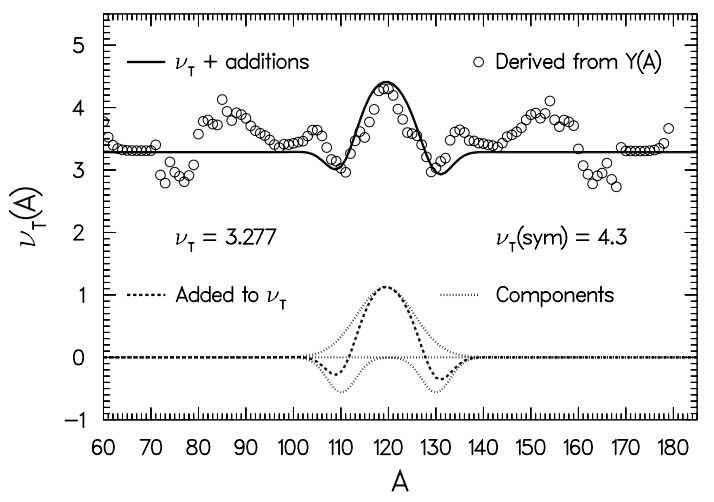

Fig. $35-\nu_{T}(\mathrm{~A})$ for Am242MT

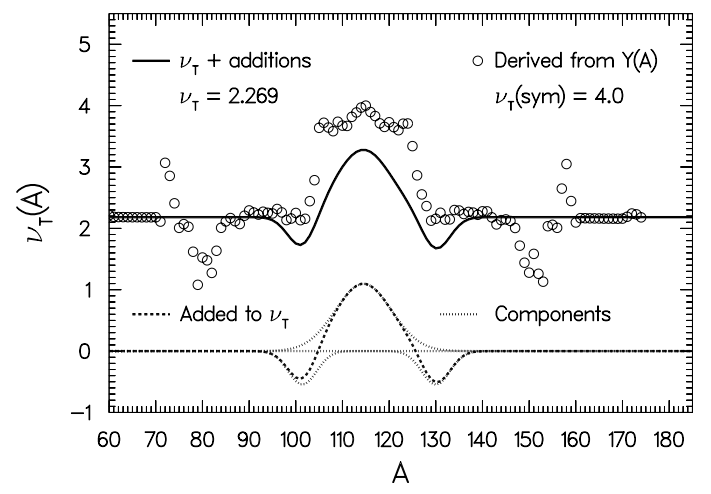

Fig. $37-\nu_{T}(\mathrm{~A})$ for $\mathrm{TH} 232 \mathrm{~F}$

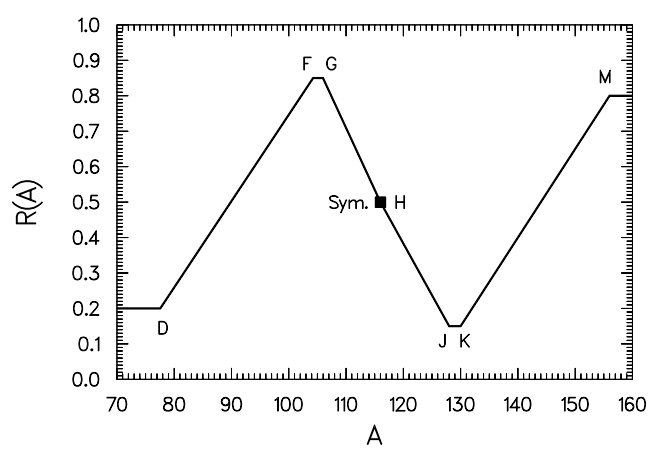

Fig. 39 - Fraction, $\mathrm{R}(\mathrm{A})$, of $\nu_{T}(\mathrm{~A})$ to A for U235T

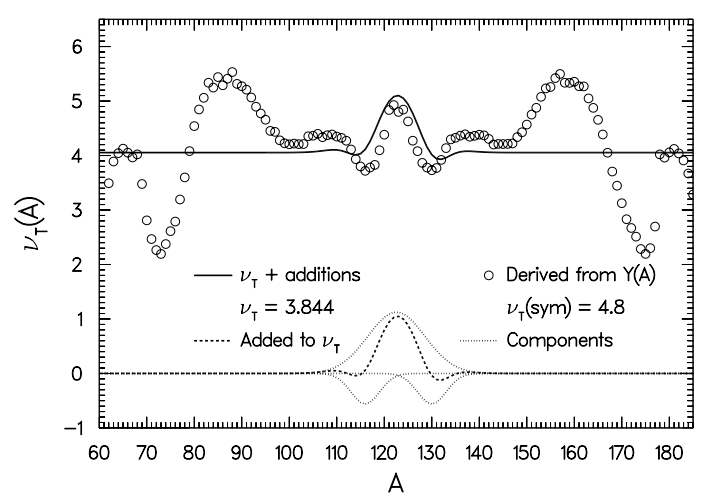

Fig. $36-\nu_{T}$ (A) for CF249T

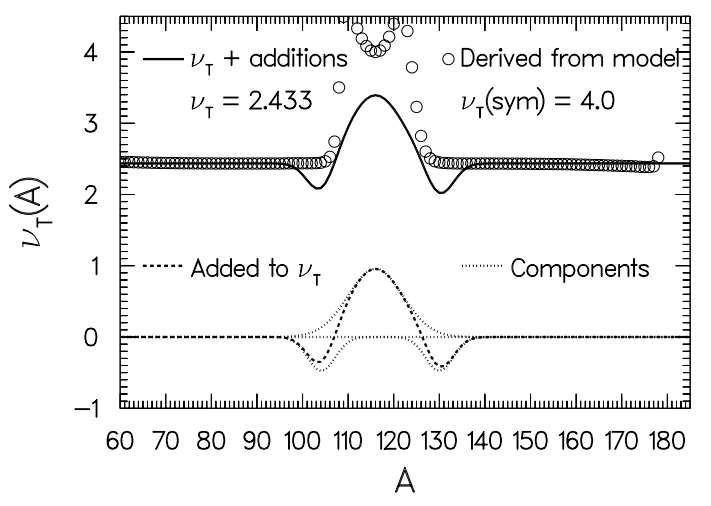

Fig. $38-\nu_{T}$ (A) for U235T, Model Y(A) Values

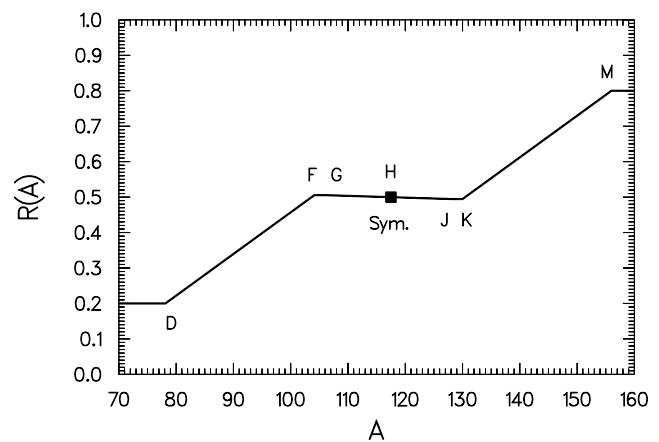

Fig. 40 -Fraction, $\mathrm{R}(\mathrm{A})$, of $\nu_{T}(\mathrm{~A})$ to $\mathrm{A}$ for ${ }^{238} \mathrm{U}+85 \mathrm{MeV} \mathrm{p}$ 


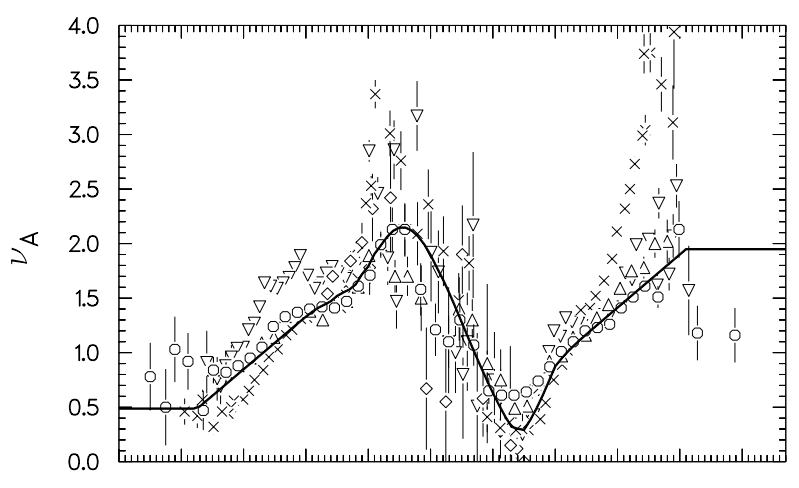

Fig. 41 -U235T, Line: Model;

Points:Experimental

(See text for references.)

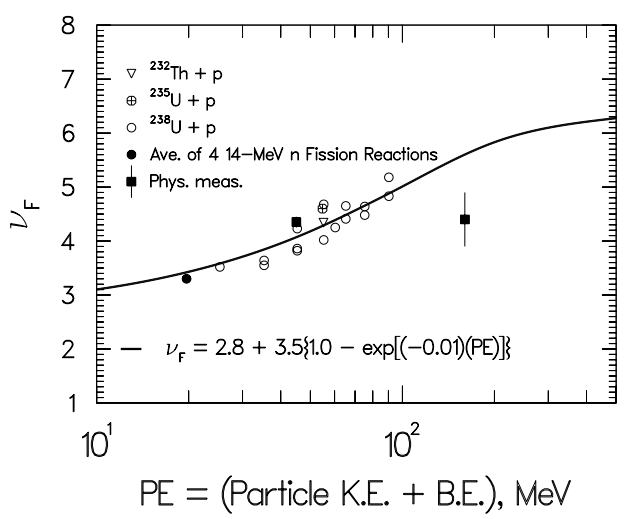

Fig. 43 - High-energy $\nu_{F}$ Function

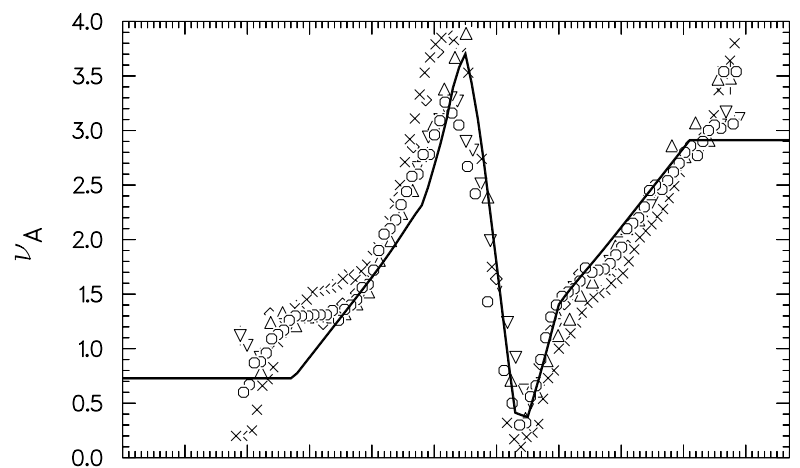

Fig. 42 -CF252S, Line:Model;

Points:Experimental

(See text for references.)

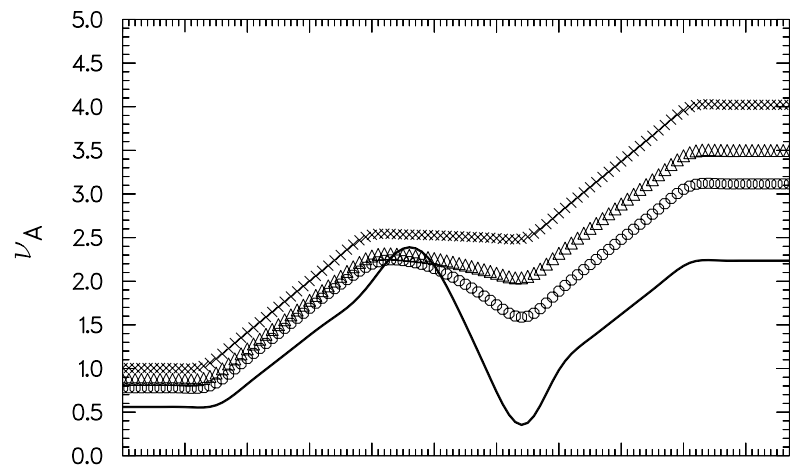

Fig. $44-\nu_{A}$ Functions, Symbols: ${ }^{238} \mathrm{U}+\rho$, o $30 \mathrm{MeV}, \Delta 50 \mathrm{MeV}$, X $85 \mathrm{MeV}$; Line: U238F 


\section{FAST-NEUTRON-INDUCED FISSION}

Neutrons from fission (fast neutrons) have a wide range of energies, so a summation calculation was carried out to determine the weighted-average value for each energy-dependent model parameter. Parameter values for each of 1,100 energy increments $0.01 \mathrm{Mev}$ wide were weighted for the relative fission cross section, $\sigma\left(E^{\prime}\right)$, and the relative yields of neutrons, $R Y\left(E^{\prime}\right)$, at the average neutron energy, $E^{\prime}$, for each energy increment. The resulting values were summed and normalized to give the weighted-average value for each energy-dependent parameter.

The relative yield, $R Y\left(E^{\prime}\right)$, was determined from Eq. 26 [LE56], the constant $C=1.29$ for U235T being multiplied by $3 / 4$ to give $C=0.9675$ to allow for energy degradation of fission-neutron-spectra neutrons in most reactor cores used for fast-neutron yield measurements.

$$
R Y\left(E^{\prime}\right)=\sqrt{E^{\prime}} e^{E^{\prime} / C}
$$

In the CYF program described next, the value of $C=V X(12)$ can be changed in the IN.PAR file to better represent actual neutron spectra, if known.

The relative cross section, $\sigma\left(E^{\prime}\right)$, which approximates experimental curves [HE63], was taken to be 1.00 over much of the energy range. The approach at low energies to $\sigma\left(E^{\prime}\right)=1.0$ depends on the fission threshold, $T H$, of fissioning nuclei with $Z_{F} \& A_{F}$. The increase of $\sigma\left(E^{\prime}\right)$ at $E_{N}=\sim 6 \mathrm{MeV}$ due to "second chance fission" depends on the fission threshold, $T H 1$, of nuclei with $Z_{F}$ \& $A_{F}-1$. The second plateau, $\sigma_{2}$, due to "first and second chance fission" can be approximated by Eq. 27, which was derived from experimental curves [HE63].

$$
\begin{gathered}
\sigma_{2}=1.533+0.093\left(A_{F}-236\right)-0.431\left(Z_{F}-92\right)+0.041\left(Z_{F}-92\right)^{2} \\
\left(\text { limits on } \sigma_{2} \text { values: } \geq 1.0, \leq 2.5\right)
\end{gathered}
$$


If $T H \leq 0.0, \sigma\left(E^{\prime}\right)$ is assumed to follow the $1 / \mathrm{v}$ law to 1.0 at $E_{N}=0.5 \mathrm{MeV}$.

$$
\sigma\left(E^{\prime}\right)=1.0+0.13 / \sqrt{E^{\prime}}-0.13 / \sqrt{0.5}
$$

If $T H>0.0, \sigma\left(E^{\prime}\right)$ is assumed to increase linearly from 0.0 at $T H$ to 1.0 at $T H+1.0$.

The second change in $\sigma\left(E^{\prime}\right)$ is assumed to increase linearly from 1.0 to $\sigma_{2}$ over $1.0 \mathrm{MeV}$ starting at the neutron binding energy, $B N$, if $T H 1 \leq 0.0$, or at $B N+T H 1$, if $T H 1>0.0$.

Errors introduced by the approximations made in expressions for $R Y\left(E^{\prime}\right)$ and $\sigma\left(E^{\prime}\right)$ are compensated, in part, in the treatment because the sum of their products are used for normalization.

For fast-neutron induced fission reactions subroutine FAST in the CYFP program has been modified to calculate the weighted-average parameter values for all energy-dependent model parameters. Thus, in addition to weighted-average values for $Y_{3}, E *$, and $\nu_{F}$, as done earlier in CYI [WA99], weighted-average values are now also calculated for $N T, \sigma_{1}, \Delta_{4}, \Delta_{5}, \sigma_{Z}, Y_{2}$, and $Y_{6}$.

\section{CALCULATION OF FISSION YIELDS FROM SYSTEMATICS - THE CYF PROGRAM}

The equations representing systematics of fission yields, which have been discussed, have been programed to create a new program, CYF, which replaces the older program, CYI. The code is written in VAX-FORTRAN, as is CYI, and formats for input and output files are the same as for the CYI program. Parameter and yield values are, of course, different. The major difference is that CYF includes equations for systematics at initial excitation energies $(P E)$ up to $\sim 200 \mathrm{MeV}$. Also, most of the equations in CYI [WA99] for yields from low-energy fission reactions have been modified to eliminate discontinuities and 
to enhance smooth transitions of the functions to those for high-energy fission reactions.

The CYF program has been modified to CYFP which can be run on a variety of computers, including the VAX and personal computers operating under Windows-98, and probably other versions. Compilation of CYFP can be accomplished using the VAX-FORTRAN compiler and/or the current version of the G77 compiler from the Internet.

As for CYI, only 4 parameters are required to run CYFP: the $A$ and $Z$ of the precursor nucleus (target + projectile, $P A$ and $P Z$ ), the type of projectile, and its kinetic energy in $\mathrm{MeV}$. Other parameters may be changed to control the calculation; some possibly useful changes are given below.

Most of the parameters needed for a calculation are in a file that is read and usually ends in .PAR (e.g., IN.PAR). In addition to a title (line 1), which is not used in the calculation but is printed at the top of most output files, there are 3 groups of parameters.

Line 2 - integer control parameters (I3)

Line 3 to $\mathrm{n}=9999$ - real parameters: $X J, V Y(J), V X(J) ;(J=X J) ;(F 4.0,2 E 13.5)$

Line $\mathrm{n}+1$ to end - constant model parameters - DO NOT CHANGE!

Line 2, col. 2-3, NR designates the projectile: 0, neutron; 10, proton; 20, deuteron; 30, triton; 40, ${ }^{3} \mathrm{He}$; 50, alpha particle.

Line 2, col. 39, ISPIN determines if isomeric-state yields are calculated: if 0 , they are not calculated; if 6 , independent yields are distributed equally among isomeric states; if 7, isomer ratios are calculated by the Madland-England method [ME77]; if 8, isomer ratios are calculated by Rudstam's method [RU92].

Line 3, col. 5-17, VY $(1)=$ precurser $A, P A ;=A_{F}$ for low energies 
Line 4, col. 5-17, $\mathrm{VY}(2)=$ precurser $Z, P Z ;=Z_{F}$ for low energies

Line 5, col. 5-17, $\mathrm{VY}(4)=$ lowest product $A$ treated, default $=60$.

Line 6, col. 5-17, VY $(5)=$ highest product $A$ treated, default $=180$.

Line 8, col. 5-17, VY $(9)=\nu_{F}$, default $=$ value from systematics; if negative, $\nu_{F}$ determined along with other energy-dependent parameters by the fast-neutron summation method described in the preceeding section.

Line 9, col. 18-30, $\mathrm{VX}(10)=$ projectile kinetic energy $(\mathrm{MeV})$, if negative, spontaneous fission is treated $\left(E^{*}=P E=0\right)$.

These and other parameters that could be changed are listed as comments at the beginning of CYFP.FOR.

There are 2 ways to run the CYFP program on a VAX. A command file may be used or the file names assigned in CYFP may be used. Only the latter option is available with a PC. The assigned file names needed are: IN.PAR, TABLE.DATH, IN.SPIN (if ISPIN >0), and IN.BR (if IBR $>0$ ). The output file names are OUT.PAR, OUT.YNU, and OUT.YLD.

\section{ACKNOWLEDGMENTS}

It is a pleasure for the author to thank Kathie Wright for preparing this manuscript and for much assistance with computer problems during the course of the investigation. Thanks are also due to Nick D'Egidio and Chuck Wilkerson for guidance in modifying CYF to CYFP and to Malcolm Fowler, Bill Inkret, and Jerry Wilhelmy for valuable comments about this report. 


\section{References}

[MC54] G. H. McCormick and B. L. Cohen. Phys. Rev., 96:722, 1954.

[LE56] R. B. Leachman. Proceedings of the international conference on peaceful uses of atomic energy. Volume 2, page 193, New York, 1956. United Nations.

[SJ56] H. M. Steiner and J. A. Jungermanm. Phys. Rev., 101:807, 1956.

[SH58] P. C. Stevenson, H. G. Hicks, W. E. Nervik and D. R. Nethaway. Phys. Rev., 111:886, 1958.

[FU59] C. B. Fulmer. Phys. Rev., 116:418, 1959.

[TE62] J. Terrell. Phys. Rev., 127:880, 1962.

[CK61] C. D. Coryell, M. Kaplin and R. D. Fink. Can. J. Chem, 39:646, 1961.

[BM63] H. R. Bowman, J. C. D. Milton, S. G. Thompson and W. J. Swiatecki. Phys. Rev., 129:2133, 1963.

[CU63] J. B. Cumming, in "Annual Review of Nuclear Science," Phys. Rev., 13:261, 1963.

[HE63] R. L. Henkel. Fission by Fast Neutrons. In J. B. Marion and J. L. Fowler, editors, Fast Neutron Physics, Part II, page 2006 and 2016. Interscience Publishers, 1963.

[BW64] H. C. Britt and S. L. Whetstone, Jr. Phys. Rev., 133:B603, 1964.

[AG65] V. F. Apalin, Yu. N. Gritsyuk, I. E. Kutikov, V. I. Lebedev and L. A. Mikaelian. Nucl. Phys., 71:553, 1965.

[BN65] Brookhaven National Laboratory Report Number 325. Neutron Cross Sections, Volume III, 2nd edition, 1965. Supplement Number 2 . 
[FL65] G. P. Ford and R. B. Leachman. Phys. Rev., 137:B226, 1965.

[MR67] E. E. Maslin, A. L. Rodgers and W. G. F. Core. Phys. Rev., 164:1520, 1967.

[CF68] E. Cheifetz and Z. Fraenkel. Phys. Rev., 21:36, 1968.

[CC69] I. F. Croall and J. G. Cunningham. Nucl. Phys., page 402, 1969. Thanks are due to Dr. Tokio Fukahori for reading values from the published curves, since data for the curves was not published.

[BV70] C. J. Bishop, R. Vandenbosch, R. Aley, R. W. Shaw Jr. and I. Halpern. Nucl. Phys., page 129, 1970.

[CF70] E. Cheifetz, A. Fraenkel, J. Galin, M. Lefort, J. Peter and X. Tarrago. Phys. Rev., page 256, 1970.

[BF70] S. C. Burnett, R. L. Ferguson, F. Plasil and H. W. Schmitt. Phys. Rev. C., 3:2034, 1970.

[BM71] J. W. Boldeman, A. R. L. Musgrove and R. L. Walsh. Aust. J. Phys., 24:821, 1971.

[MK72] F. Manero and V. A. Kinshin. Atomic Energy Review, volume 10, page 637. International Atomic Energy Agency, Vienna, 1972.

[TC72] B. L. Tracy, J. Chaumont, R. Klapish, J. M. Nitschke, A. M. Poskanzer, E. Roeckl and C. Thibault. Phys. Rev. C., 5:222, 1972.

[SB73] C. Signarbieux, R. Babinet, H. Nifenecker and J. Poitou. Physics and Chemistry of Fission, page 179. International Atomic Energy Agency, Vienna, 1973.

[VH73] R. Vandenbosch and J. R. Huizenga. Nuclear Fission. Academic Press, New York, 1973.

[DM74] M. Diksic, D. K. McMillan and L. Yaffe. J. Inorg. Nucl. Chem., 36:7, 1974. 
[MC74] A. R. deL. Musgrove, J. L. Cook and G. D. Trimble. Proceedings of a panel on fission product nuclear data. In Proc. Fifteenth Annual $A C M$, number Vol. II in IAEA-169, page 163, Bologna, 1974 Vienna.

[NE74] D. R. Nethaway. Ucrl-51538. Technical report, 1974.

[SY76] S. Sarkar and L. Yaffe. Can. J. Chem., 54:2349, 1976.

[HO77] R. J. Howerton. Nuc. Sci. Eng., 62:438, 1977.

[ME77] D. G. Madland and T. R. England. Nuc. Sci. Eng., 64:859, 1977.

[WB77] R. L. Walsh and J. W. Boldeman. Nucl. Phys., pages 276, 189, 1977.

[DI78] J. K. Dickins. Nucl. Sci. Eng., 96:8, 1978.

[NF78] S. Nagy, K. F. Flynn, J. E. Gindler, J. W. Meadows and L. E. Glendenin. Phys. Rev. C., 17, 1978.

[GI79] J. Gindler. Phys. Rev. C., 19:1806, 1979.

[GG80] L. E. Glendenin, J. E. Gindler, I. Ahmad, D. J. Henderson and J. W. Meadows. Phys. Rev. C., 22(1):152, 1980.

[NP80] L. Nikkinen, B. P. Pathak, L. Lessard and I. S. Grant. Phys. Rev. C., 22:617, 1980 .

[GG81] L. E. Glendenin, J. E. Gindler, D. J. Henderson and J. W. Meadows. Phys. Rev. C., 24(6):2600, 1981.

[CH82] C. Chung and J. J. Hogan. Phys. Rev. C., 25,899, 1982.

[BB83] D. Belhafaf, J.P. Bocquet, R. Brissot, Ch. Ristori, J. Crancon, H. Nifenecker, J. Mougey, and V. S. Ramamurthy. Z. Phys. A., 309:253, 1983.

[GG83] J. E. Gindler, L. E. Glendenin, D. J. Henderson and J. W. Meadows. Phys. Rev. C., page 2058, 1983. 
[SH83] R. Schmidt and H. Henschel. Nucl. Phys., page 29, 1983.

[AX84] E. J. Axton. European Applied Research Report - Nuclear Science and Technology. Number Vol. 5, page 609, 1984.

[MN84] R. Miller, A. A. Naqvi, F. Kaeppeler and F. Dickmann. Phys. Rev. C., 29:885, 1984 .

[DW86] A. J. Deruytter, C. Wagemans, F. Corvi, E. J. Axton, and P. Schillebeeckx. Gelph Conference. 1986.

[WA88] A. C. Wahl. Atomic Data and Nuclear Data Tables, 39:1, 1988.

[DA89] M. Djebara, M. Asghar, J. P. Bocquet, R. Brissot, J. Crancon, Ch. Ristori, E. Aker, D. Engelhardt, J.Gindler, B. D. Wilkins, U. Quade and K. Rudolph. Nucl. Phys., A496:346, 1989.

[SA89] J. L. Sida, P. Armbruster, M. Bernas, J. P. Bocquet, R. Brissot and H. R. Faust. Nucl. Phys., A502:233, 1989.

[WA89] A. C. Wahl, in "50 Years with Nuclear Fission," Volume 2, page 525. American Nuclear Society, La Grange Park, Illinois, 1989. presented at the conference on 50 Years with Nuclear Fission, Washington D. C., April 25-28, 1989.

[DI91] W. Ditz. ${ }^{239} \mathrm{Pu}(n$ th, f) Light Wing Fission Yields. Thesis, Institut fur Kernschemie, Guttenberg-Universitat Mainz, D-6500 Mainz, Germany, 1991.

[JM91] M. F. James, R. W. Mills and D. R. Weaver. Reports aea-trs-1015. Technical report, 1991.

[RU92] G. Rudstam. Personal communication from Professor Rudstam. 1992.

[WA92] A. C. Wahl. Proceedings of a Specialists' Meeting on Fission Product Nuclear Data. In Proc. Fifteenth Annual ACM, page 334, TokaiMura, Ibaraka-Ken, Japan, May 25-27 1992. Los Alamos National 
Laboratory Report No. LA-UR-92-1425 also contains tables of parameter values for empirical models in 3 pages of Appendices.

[ER93] T. R. England and B. F. Rider. Los Alamos National Laboratory Report LAUR-94-3106, ENDF. Technical Report, 1993.

[HE94] R. Hentzschel, H. R. Faust, H. O. Denschlag, B. D. Wilkins, and J. Gindler. Nucl. Phys., page 427, 1994.

[DE95] H. O. Denschlag. Personal communication from Professor Denschlag. 1995.

[MI95] R. W. Mills. Fission product yield evaluation. PhD thesis, University of Birmingham, U. K., 1995.

[MS96] M. Mutterer and P. P. Sieger. Personal communication. 1996.

[ST96] P. Stumpf. Thesis Concerning ${ }^{241}$ Am(2nth, f) Light Wing Fission Yields. Thesis, Guttenberg-Universitat Mainz, D-6500 Mainz, Germany, 1996.

[WA99] A. C. Wahl. Compilation and evaluation of fission yield nuclear data, pages 45-75, 169, 170, Appendicies (CD-ROM) A.1, B.1. International Atomic Energy Agency, Vienna, 2000. Contributions to the International Atomic Energy Agency Report IAEA-TECDOC1168 . 

This report has been reproduced directly from the best available copy. It is available electronically on the Web (http://www.doe.gov/bridge).

Copies are available for sale to U.S. Department of Energy employees and contractors from-

Office of Scientific and Technical Information

P.O. Box 62

Oak Ridge, TN 37831

(865) 576-8401

Copies are available for sale to the public from-

National Technical Information Service

U.S. Department of Commerce

5285 Port Royal Road

Springfield, VA 22616

(800) 553-6847 


\section{$\overbrace{\text { LATIONAL LAB ORATORY }}$}

Los Alamos NM 87545 